\title{
UNIVERSAL ASSOCIATIVE ENVELOPES OF NONASSOCIATIVE TRIPLE SYSTEMS
}

\author{
HADER A. ELGENDY
}

\begin{abstract}
We construct universal associative envelopes for the nonassociative triple systems arising from the trilinear operations of Bremner and Peresi applied to the 2-dimensional simple associative triple system. We use noncommutative Gröbner bases to determine monomial bases, structure constants, and centers of the universal envelopes. We show that the infinite dimensional envelopes are closely related to the down-up algebras of Benkart and Roby. For the finite dimensional envelopes, we determine the Wedderburn decompositions and classify the irreducible representations.
\end{abstract}

\section{INTRODUCTION}

In this paper we use noncommutative Gröbner bases to construct the universal associative enveloping algebras of the nonassociative triple systems which arise from applying the nonassociative trilinear operations classified by Bremner and Peresi 5 to the 2-dimensional simple associative triple system of the first kind in the sense of Lister [17, namely the space of $2 \times 2$ matrices $A=\left(a_{i j}\right)$ with $a_{11}=a_{22}=0$.

The basic goal of this work is reduce the problem of representation of these triple systems, which are the simplest examples of nonassociative triple systems satisfying polynomial identities in degree 3 and 5 to that of associative algebras. They provide natural generalizations of Lie and Jordan triple systems, and therefore have the potential for a wide range of applications to various branches of mathematics, especially to the theory of Lie groups and non-Euclidean geometry [11, 12, 21]. Indeed, they have arisen in the study of symmetric spaces [18, and have been connected with the study of the Yang-Baxter equations [16].

By a multilinear $n$-ary operation we mean an element $\omega$ of the group algebra $\mathbb{Q} S_{n}$ of the symmetric group $S_{n}$ over the rational field $\mathbb{Q}$. Following [5] we say that two operations $\omega_{1}, \omega_{2}$ are equivalent if they generate the same left ideal. If $A$ is an associative algebra over $\mathbb{Q}$, then $\omega$ defines a multilinear $n$-ary operation $\omega\left(a_{1}, \ldots, a_{n}\right)$ on the underlying vector space of $A$ :

$$
\omega=\sum_{\sigma \in S_{n}} x_{\sigma} \sigma \quad \Longrightarrow \quad \omega\left(a_{1}, \ldots, a_{n}\right)=\sum_{\sigma \in S_{n}} x_{\sigma} a_{\sigma(1)} \cdots a_{\sigma(n)} .
$$

In this way we obtain a nonassociative $n$-ary algebra which we denote by $A^{\omega}$.

For $n=2$, every bilinear operation is equivalent to either the zero operation, the associative operation $a b$, the Lie bracket $[a, b]=a b-b a$, or the Jordan product $a \circ b=\frac{1}{2}(a b+b a)$. The polynomial identities of degree $\leq 3(\leq 4)$ satisfied by the Lie bracket (Jordan product) define Lie algebras (Jordan algebras), the most important varieties of nonassociative algebras. For $n=3$, Bremner and Peresi [5] found canonical representatives of the equivalence classes of trilinear operations, and identified 19 operations satisfying polynomial identities of degree 5 which do 


$\begin{array}{ll}\text { symmetric sum } & a(b \circ c)+b(c \circ a)+c(a \circ b) \\ \text { alternating sum } & a[b, c]+b[c, a]+c[a, b] \\ \text { cyclic sum } & a b c+b c a+c a b \\ \text { Lie family } q=\infty & {[a,[b, c]]} \\ \text { Lie family } q=\frac{1}{2} & {[a, b \circ c]} \\ \text { Jordan family } q=\infty & a b c+c b a \\ \text { Jordan family } q=0 & (a \circ b) c \\ \text { Jordan family } q=\frac{1}{2} & a(b \circ c)+c(a \circ b)+(c \circ a) b \\ \text { Jordan family } q=1 & a(b \circ c) \\ \text { anti-Jordan family } q=\infty & a[b, c]+c[a, b]+[c, a] b \\ \text { anti-Jordan family } q=-1 & a[b, c] \\ \text { anti-Jordan family } q=\frac{1}{2} & a b c-c b a \\ \text { anti-Jordan family } q=2 & {[a, b] c} \\ \text { fourth family } q=\infty & a b c-a c b-b a c \\ \text { fourth family } q=0 & a b c-a c b+b c a \\ \text { fourth family } q=1 & a b c-b a c+c a b \\ \text { fourth family } q=-1 & a b c+b a c+c a b \\ \text { fourth family } q=2 & a b c+a c b+b c a \\ \text { fourth family } q=\frac{1}{2} & {[a b c+a c b+b a c} \\ \text { cyclic commutator } & \\ \text { weakly commutative } & \left\{\begin{array}{l}a b c+a c b+b a c-c b a, \\ a b c-a c b+2 b a c\end{array}\right. \\ \text { weakly anticommutative } & \left\{\begin{array}{l}a b c+a c b-b a c-c b a, \\ a b c+a c b-2 b a c\end{array}\right. \\ & \end{array}$

TABLE 1. The twenty-two trilinear operations

not follow from the identities of degree 3. These operations include the Lie, anti-Lie, Jordan, and anti-Jordan triple products.

In Section 2] we find simpler operations equivalent to those of [5]; our operations have coefficients $\pm 1, \pm 2$ and most have coefficients \pm 1 . We augment this list with the symmetric, alternating and cyclic sums; see Table 1

In Section 3 we recall the theory of noncommutative Gröbner bases for ideals in free associative algebras, and use it to define universal associative envelopes $U\left(A^{\omega}\right)$ of nonassociative $n$-ary algebras $A^{\omega}$ defined by multilinear operations $\omega$.

In Section 4 we recall the down-up algebras of Benkart and Roby 3 . We then consider the cases in which $U\left(A^{\omega}\right)$ is infinite dimensional: we determine monomial bases and structure constants, identify the center, and determine the GelfandKirillov dimension. In every case, $U\left(A^{\omega}\right)$ is either a free associative algebra, a down-up algebra, or a quotient of a down-up algebra.

In Section [5 we consider the cases in which $U\left(A^{\omega}\right)$ is finite dimensional. We use an algorithmic version of the structure theory for finite dimensional associative algebras to determine the Wedderburn decompositions and classify the irreducible representations. In most cases we obtain only the trivial 1-dimensional representation and the natural 2-dimensional representation.

The results of Sections 4 and 5 are summarized in Table 2 We distinguish trilinear operations of "Lie type" for which $U\left(A^{\omega}\right)$ is infinite dimensional, and those of "Jordan type" for which $U\left(A^{\omega}\right)$ is finite dimensional. Recall that for a 
finite dimensional Lie algebra $L$, the universal associative envelope $U(L)$ is infinite dimensional, and the map $L \rightarrow U(L)$ is injective; whereas for a finite dimensional Jordan algebra $J$, the universal associative envelope $U(J)$ is finite dimensional, and the map $J \rightarrow U(J)$ is injective if and only if $J$ is special.

For the cases where the universal associative envelope is finite dimensional, as in the representation theory of Jordan algebras and triple systems [15, 19, 20, we obtain a complete description of the Wedderburn decomposition of the universal associative envelope, and this provides a complete classification of the finite dimensional irreducible representation of the triple system. These cases also provide natural examples where the computational approach to the Wedderburn decomposition can be applied. For the cases where the universal associative envelope is infinite dimensional, as in the representation theory of Lie algebras and triple systems [13, we provide a monomial basis for the envelope and the structures constants with respect to this basis. These cases are closely related to the theory of down-up algebras, and provide a natural way to generalize down-up algebras to structures with more than two generators and/or defined by relations of degree greater than 3. These cases also provide natural generalizations of the enveloping algebras of Lie algebras, and may therefore be connected to the theory of quantum groups [7.

We assume throughout that the base field $\mathbb{F}$ has characteristic 0 ; if necessary, we assume that $\mathbb{F}$ is algebraically closed.

\section{ThE TWENTY-TWO TRILINEAR OPERATIONS}

A natural basis for $\mathbb{Q} S_{3}$ consists of the six permutations in lexicographical order: $\{a b c, a c b, b a c, b c a, c a b, c b a\}$. Another natural basis consists of the matrix units $\left\{S, E_{11}, E_{12}, E_{21}, E_{22}, A\right\}$ for the decomposition as a direct sum $\mathbb{Q} \oplus M_{2}(\mathbb{Q}) \oplus \mathbb{Q}$ of simple ideals corresponding to the partitions $3=2+1=1+1+1$ which label the irreducible representations of $S_{3}$. Bremner and Peresi [5] give the matrix $M$ whose columns express the matrix units as linear combinations of the permutations:

$$
M=\frac{1}{6}\left[\begin{array}{rrrrrr}
1 & 2 & 0 & 0 & 2 & 1 \\
1 & 0 & 2 & 2 & 0 & -1 \\
1 & 2 & -2 & 0 & -2 & -1 \\
1 & -2 & 2 & -2 & 0 & 1 \\
1 & 0 & -2 & 2 & -2 & 1 \\
1 & -2 & 0 & -2 & 2 & -1
\end{array}\right], \quad M^{-1}=\left[\begin{array}{rrrrrr}
1 & 1 & 1 & 1 & 1 & 1 \\
1 & 0 & 1 & 0 & -1 & -1 \\
0 & 1 & 0 & 1 & -1 & -1 \\
0 & 1 & -1 & -1 & 1 & 0 \\
1 & 0 & -1 & -1 & 0 & 1 \\
1 & -1 & -1 & 1 & 1 & -1
\end{array}\right] .
$$

Given a trilinear operation $\omega=x_{1} a b c+x_{2} a c b+x_{3} b a c+x_{4} b c a+x_{5} c a b+x_{6} c b a$ with coefficient vector $X=\left[x_{1}, x_{2}, x_{3}, x_{4}, x_{5}, x_{6}\right]^{t}$, one obtains its matrix form,

$$
Y=\left[y_{1},\left[\begin{array}{ll}
y_{2} & y_{3} \\
y_{4} & y_{5}
\end{array}\right], y_{6}\right],
$$

by $Y=M^{-1} X$. Two operations are equivalent if and only if their matrix forms are row-equivalent; hence canonical representatives of the equivalence classes are the operations for which each component matrix is in row canonical form.

To find the simplest representative of each equivalence class, we first consider the $3^{5}=243$ operations whose coefficients in the permutation basis are $\left[1, x_{2}, \ldots, x_{6}\right]$ where $x_{2}, \ldots, x_{6} \in\{1,0,-1\}$. We record the operations whose matrix forms are among the canonical representatives [5]; this gives 20 of the 22 operations. We next consider the $5^{5}=3125$ operations whose coefficients in the permutation basis 
Operations of Lie type

\begin{tabular}{|c|c|c|c|}
\hline & $\operatorname{dim} U\left(A^{\omega}\right)$ & GK-dim & $U\left(A^{\omega}\right)$ \\
\hline$\left\{\begin{array}{l}\text { symmetric sum } \\
\text { cyclic sum }\end{array}\right.$ & $\infty$ & 1 & $A(-1,-1,1) /\left\langle a^{3}, b^{3}\right\rangle$ \\
\hline alternating sum & $\infty$ & $\infty$ & $F\langle a, b\rangle$ \\
\hline$\left\{\begin{array}{l}\text { Lie } q=\infty \\
\text { anti-Jordan } q=\infty\end{array}\right.$ & $\infty$ & 3 & $A(2,-1,-2)$ \\
\hline$\left\{\begin{array}{l}\text { Lie } q=\frac{1}{2} \\
\text { anti-Jordan } q=\frac{1}{2}\end{array}\right.$ & $\infty$ & 3 & $A(0,1,0)$ \\
\hline \multicolumn{4}{|l|}{ Operations of Jordan type } \\
\hline \multirow{3}{*}{$\begin{array}{l}\text { Jordan } q=\infty \\
\text { Jordan } q=\frac{1}{2} \\
\text { anti-Jordan } q=-1 \\
\text { anti-Jordan } q=2 \\
\text { fourth family } q=\infty \\
\text { fourth family } q=-1 \\
\text { fourth family } q=2 \\
\text { fourth family } q=\frac{1}{2} \\
\text { cyclic commutator } \\
\text { weakly commutative } \\
\text { weakly anticommutative }\end{array}$} & $\operatorname{dim} U\left(A^{\omega}\right)$ & GK-dim & $U\left(A^{\omega}\right)$ \\
\hline & & & \\
\hline & 5 & 0 & $\mathbb{Q} \oplus M_{2 \times 2}$ \\
\hline$\left\{\begin{array}{l}\text { Jordan } q=0 \\
\text { fourth family } q=0\end{array}\right.$ & 9 & 0 & $\mathfrak{R} \oplus \mathbb{Q} \oplus M_{2 \times 2}$ \\
\hline$\left\{\begin{array}{l}\text { Jordan } q=1 \\
\text { fourth family } q=1\end{array}\right.$ & 9 & 0 & $\mathfrak{R} \oplus \mathbb{Q} \oplus M_{2 \times 2}$ \\
\hline
\end{tabular}

TABLE 2. Structure of the universal associative envelopes

satisfy $x_{2}, \ldots, x_{6} \in\{2,1,0,-1,-2\}$; this gives the remaining two operations, and also produces alternative forms of the last two operations. In more than half of the cases, the simplified operations of Table 1 are more natural than the original operations, since they can be easily expressed in terms of the Lie bracket and the Jordan product.

\section{Gröbner BASES AND UNIVERSAL ENVELOPES}

We first recall basic results about noncommutative Gröbner bases for ideals in free associative algebras. Standard references are Bergman [1], de Graaf [8]; for another application to $n$-ary algebras, see Elgendy and Bremner [9].

Definition 3.1. Let $X=\left\{x_{1}, \ldots, x_{n}\right\}$ be a set of symbols with total order $x_{i}<x_{j}$ $\Longleftrightarrow i<j$. The free monoid $X^{*}$ on $X$ consists of all (possibly empty) words $w=x_{i_{1}} \cdots x_{i_{k}}(k \geq 0)$ with the associative operation of concatenation. For $w=$ $x_{i_{1}} \cdots x_{i_{k}} \in X^{*}$ the degree is $\operatorname{deg}(w)=k$. The free unital associative algebra $F\langle X\rangle$ is the vector space with basis $X^{*}$ and multiplication extended bilinearly. The deglex (degree-lexicographical) order on $X^{*}$ is defined by: $u<v$ if and only if either $(i)$ 
$\operatorname{deg}(u)<\operatorname{deg}(v)$ or $(i i) \operatorname{deg}(u)=\operatorname{deg}(v)$ and $u=w x_{i} u^{\prime}, v=w x_{j} v^{\prime}$ where $x_{i}<x_{j}$ $\left(w, u^{\prime}, v^{\prime} \in X^{*}\right)$. We say that $u \in X^{*}$ is a factor of $v \in X^{*}$ if there exist $w_{1}, w_{2} \in X^{*}$ such that $w_{1} u w_{2}=v$. If $w_{1}\left(w_{2}\right)$ is empty then $u$ is a left (right) factor of $v$.

Definition 3.2. The support of $f \in F\langle X\rangle$ is the set of monomials $w \in X^{*}$ that occur in $f$ with nonzero coefficient. The leading monomial $\operatorname{LM}(f)$ is the greatest element of the support of $f$. For an ideal $I \subseteq F\langle X\rangle$ the set of normal words modulo $I$ is $N(I)=\left\{u \in X^{*} \mid u \neq \operatorname{LM}(f)\right.$ for any $\left.f \in I\right\}$, and $C(I)$ is the subspace of $F\langle X\rangle$ with basis $N(I)$.

Proposition 3.3. We have $F\langle X\rangle=C(I) \oplus I$.

Definition 3.4. Let $G \subset F\langle X\rangle$ be a subset generating an ideal $I$. An element $f \in F\langle X\rangle$ is in normal form modulo $G$ if no monomial in the support of $f$ has $\operatorname{LM}(g)$ as a factor for any $g \in G$.

Definition 3.5. A subset $G \subset I$ is a Gröbner basis of $I$ if for every $f \in I$ there exists $g \in G$ such that $\operatorname{LM}(g)$ is a factor of $\operatorname{LM}(f)$.

Definition 3.6. A subset $G \subset F\langle X\rangle$ is self-reduced if every $g \in G$ is in normal form modulo $G \backslash\{g\}$ and every $g \in G$ is monic: the coefficient of $\operatorname{LM}(g)$ is 1 .

Definition 3.7. Consider elements $g, h \in F\langle X\rangle$ such that $\operatorname{LM}(g)$ is not a factor of $\operatorname{LM}(h)$ and $\operatorname{LM}(h)$ is not a factor of $\operatorname{LM}(g)$. Assume that $u, v \in X^{*}$ satisfy $\operatorname{LM}(g) u=v \operatorname{LM}(h), u$ is a proper right factor of $\operatorname{LM}(h)$, and $v$ is a proper left factor of $\operatorname{LM}(g)$. Then $g u-v h$ is called a composition of $g$ and $h$.

Theorem 3.8. If $I \subset F\langle X\rangle$ is an ideal generated by a self-reduced set $G$, then $G$ is a Gröbner basis of $I$ if and only if for all compositions $f$ of the elements of $G$ the normal form of $f$ modulo $G$ is zero.

In order to compute a Gröbner basis for an ideal $I \subset F\langle X\rangle$, we start with any set $G$ of generators for $I$, and perform the following algorithm:

(1) Compute all compositions of elements of $G$. Let $H$ be the set of their normal forms modulo $G$.

(2) Replace $G$ by $G \cup H$, and self-reduce the new set $G$ by replacing each element by its normal form modulo the other elements.

The algorithm terminates when $H=\emptyset$ or $H=\{0\}$.

We now apply this theory to the construction of universal associative envelopes. Given a finite dimensional associative algebra $A$, and a multilinear $n$-ary operation $\omega$, we obtain a nonassociative $n$-ary algebra $A^{\omega}$.

Definition 3.9. A universal associative envelope of $A^{\omega}$ consists of a unital associative algebra $U\left(A^{\omega}\right)$ and a linear map $i: A^{\omega} \rightarrow U\left(A^{\omega}\right)$ satisfying

$$
i\left(\omega\left(x_{1}, x_{2}, \ldots, x_{n}\right)\right)=\omega\left(i\left(x_{1}\right), i\left(x_{2}\right), \ldots, i\left(x_{n}\right)\right),
$$

for all $x_{1}, \ldots, x_{n} \in A^{\omega}$, such that for any unital associative algebra $\mathbb{A}$ and linear map $j: A^{\omega} \rightarrow \mathbb{A}$ satisfying the same equation with $j$ in place of $i$, there is a unique homomorphism of unital associative algebras $\psi: U\left(A^{\omega}\right) \rightarrow \mathbb{A}$ such that $\psi \circ i=j$.

Notation 3.10. Let $B=\left\{e_{1}, e_{2}, \ldots, e_{m}\right\}$ be an ordered basis of $A^{\omega}$, and let $\phi: B \rightarrow X=\left\{x_{1}, x_{2}, \ldots x_{m}\right\}$ be the bijection $\phi\left(e_{i}\right)=x_{i}$. We extend $\phi$ to a linear map, denoted by the same symbol, $\phi: A^{\omega} \rightarrow F\langle X\rangle$. 
Definition 3.11. Consider the following elements of $F\langle X\rangle$ :

$$
G_{i_{1}, \ldots, i_{n}}=\omega\left(x_{i_{1}}, \ldots, x_{i_{n}}\right)-\phi\left(\omega\left(e_{i_{1}}, \ldots, e_{i_{n}}\right)\right), \quad 1 \leq i_{1}, \ldots, i_{n} \leq m .
$$

Let $I$ be the ideal generated by the set of all $G_{i_{1}, \ldots, i_{n}}$, and define $U\left(A^{\omega}\right)=F\langle X\rangle / I$. We have the natural surjection $\pi: F\langle X\rangle \rightarrow U\left(A^{\omega}\right)$ sending $f$ to $f+I$, and the composition $i=\pi \circ \phi: A^{\omega} \rightarrow U\left(A^{\omega}\right)$.

Lemma 3.12. The algebra $U\left(A^{\omega}\right)$ and the map $i: A^{\omega} \rightarrow U\left(A^{\omega}\right)$ form the universal associative envelope of the nonassociative $n$-ary algebra $A^{\omega}$.

To obtain the elements $G_{i_{1}, \ldots, i_{n}}$, we use the structure constants of $A^{\omega}$. We then use Theorem 3.8 to compute a Gröbner basis of the ideal $I$, and Proposition 3.3 to determine a basis of $U\left(A^{\omega}\right)$.

Notation 3.13. We write $\delta_{i, j}$ for the Kronecker delta, and $\widehat{\delta}_{i, j}=1-\delta_{i, j}$.

\section{INFINITE DIMENSIONAL ENVELOPES}

In this section, we consider the trilinear operations of "Lie type".

Definition 4.1. (Benkart and Roby [3) Let $\mathbb{F}$ be a field and let $\alpha, \beta, \gamma \in \mathbb{F}$ be parameters. The down-up algebra $A(\alpha, \beta, \gamma)$ is the unital associative algebra with generators $a, b$ and relations

$$
b^{2} a=\alpha b a b+\beta a b^{2}+\gamma b, \quad b a^{2}=\alpha a b a+\beta a^{2} b+\gamma a .
$$

Theorem 4.2. [3, Theorem 3.1, Corollary 3.2] A down-up algebra has basis

$$
\mathfrak{B}_{1}=\left\{a^{i}(b a)^{j} b^{k} \mid i, j, k \geq 0\right\},
$$

and its Gelfand-Kirillov dimension is 3.

Lemma 4.3. 23, Lemma 2.2] For any $c_{1}, c_{2} \in \mathbb{F}$, a down-up algebra has basis

$$
\mathfrak{B}_{2}=\left\{a^{i}\left(b a+c_{1} a b+c_{2}\right)^{j} b^{k} \mid i, j, k \geq 0\right\} .
$$

For the rest of this paper, $A$ is the associative triple system with basis

$$
e_{1}=\left[\begin{array}{ll}
0 & 1 \\
0 & 0
\end{array}\right], \quad e_{2}=\left[\begin{array}{ll}
0 & 0 \\
1 & 0
\end{array}\right]
$$

We make the underlying vector space of $A$ into a nonassociative triple system $A^{\omega}$ in different ways corresponding to the trilinear operations $\omega$ of Table 1 . Let $X=\{a, b\}$ with $a<b$, and define $\phi: A^{\omega} \rightarrow F\langle X\rangle$ by $\phi\left(e_{1}\right)=a, \phi\left(e_{2}\right)=b$.

4.1. The symmetric sum. The structure constants for $A^{\omega}$ are determined by

$$
\left[e_{1}, e_{1}, e_{1}\right]=\left[e_{2}, e_{2}, e_{2}\right]=0, \quad\left[e_{2}, e_{1}, e_{1}\right]=2 e_{1}, \quad\left[e_{1}, e_{2}, e_{2}\right]=2 e_{2} .
$$

Lemma 4.4. A basis for $U\left(A^{\omega}\right)$ is the set $\left\{a^{i}(b a)^{j} b^{k} \mid 0 \leq i, k \leq 2, j \geq 0\right\}$.

Proof. We have $U\left(A^{\omega}\right)=F\langle a, b\rangle / I$ where $I$ is generated by $G=\left\{G_{1}, G_{2}, G_{3}, G_{4}\right\}$ :

$$
G_{1}=b^{3}, \quad G_{2}=b^{2} a+b a b+a b^{2}-b, \quad G_{3}=b a^{2}+a b a+a^{2} b-a, \quad G_{4}=a^{3} .
$$

We show that the set $G$ is a Gröbner basis of $I$. There are seven compositions:

$$
\begin{aligned}
& S_{1}=G_{1} a-b G_{2}, \quad S_{2}=G_{1} b a-b^{2} G_{2}, \quad S_{3}=G_{1} a^{2}-b^{2} G_{3}, \quad S_{4}=G_{2} a^{2}-b^{2} G_{4}, \\
& S_{5}=G_{2} a-b G_{3}, \quad S_{6}=G_{3} a^{2}-b a G_{4}, \quad S_{7}=G_{3} a-b G_{4} .
\end{aligned}
$$


We eliminate from $S_{1}, \ldots, S_{7}$ all occurrences of the leading monomials of $G_{1}, G_{2}$, $G_{3}, G_{4}$; we write $\equiv$ to indicate congruence modulo $G$ :

$$
\begin{aligned}
S_{1}= & -b^{2} a b-b a b^{2}+b^{2} \equiv-\left(-b a b-a b^{2}+b\right) b-b a b^{2}+b^{2} \equiv 0, \\
S_{2}= & -b^{2}\left(b a b+a b^{2}-b\right) \equiv-\left(-b a b-a b^{2}+b\right) b^{2} \equiv 0, \\
S_{3}= & -b^{2} a b a-b^{2} a^{2} b+b^{2} a=-b^{2} a(b a+a b-1) \\
\equiv & -\left(-b a b-a b^{2}+b\right)(b a+a b-1) \\
\equiv & b a\left(-b a b-a b^{2}+b\right)+b a b a b-b a b+a\left(-b a b-a b^{2}+b\right) b-a b^{2} \\
& +b a b+a b^{2}-b-b a b+b=-b a^{2} b^{2}+a\left(-b a b-a b^{2}+b\right) b \\
\equiv & \left(a b a+a b^{2}-a\right) b^{2}-a b a b^{2}+a b^{2} \equiv 0, \\
S_{4}= & b a b a^{2}+a b^{2} a^{2}-b a^{2} \equiv b a\left(-a b a-a^{2} b\right)+a\left(-b a b-a b^{2}+b\right) a \\
\equiv & -\left(-a b a-a^{2} b+a\right) b a+a\left(-b a b-a b^{2}+b\right) a \equiv 0 .
\end{aligned}
$$

Similar calculations show that $S_{5}, S_{6}, S_{7} \equiv 0$. Hence $G$ is a Gröbner basis of $I$, and Proposition 3.3 completes the proof.

Corollary 4.5. In $U\left(A^{\omega}\right)$, we have the relations

$$
a^{3}=b^{3}=0, \quad b^{2} a=-b a b-a b^{2}+b, \quad b a^{2}=-a b a-a^{2} b+a .
$$

Hence $U\left(A^{\omega}\right)$ is the quotient of $A(-1,-1,1)$ by the ideal generated by $a^{3}$ and $b^{3}$.

Definition 4.6. Consider the anti-automorphism $\zeta: F\langle a, b\rangle \rightarrow F\langle a, b\rangle$ defined by $\zeta(a)=b$ and $\zeta(b)=a$. Since $\zeta\left(G_{4}\right)=G_{1}, \zeta\left(G_{1}\right)=G_{4}, \zeta\left(G_{2}\right)=G_{3}, \zeta\left(G_{3}\right)=G_{2}$, we see that $\zeta$ induces an anti-automorphism on $U\left(A^{\omega}\right)$, also denoted $\zeta$.

A filtration $\{0\} \subseteq V^{(0)} \subseteq V^{(1)} \subseteq \cdots \subseteq \bigcup_{n} V^{(n)}=U\left(A^{\omega}\right)$ is defined by letting $V^{(n)}$ be the subspace with basis consisting of all $a^{i}(b a)^{j} b^{k}$ where $0 \leq i, k \leq 2, j \geq 0$, and $i+2 j+k \leq n$. The associated graded algebra is

$$
\operatorname{gr}\left(U\left(A^{\omega}\right)\right)=\bigoplus_{i \geq 0} \mathcal{G}^{i}\left(U\left(A^{\omega}\right)\right), \quad \mathcal{G}^{i}\left(U\left(A^{\omega}\right)\right)=V^{(i)} / V^{(i-1)}, \quad V^{-1}=\{0\} .
$$

Corollary 4.7. The dimension of $\mathcal{G}^{n}\left(U\left(A^{\omega}\right)\right)$ is 1 if $n=0,2$ if $n=1,4$ if $n=2$ or $n \geq 3$ (odd), and 5 if $n \geq 4$ (even).

Proof. For $n=0$, there is one monomial: $(i, j, k)=(0,0,0)$. For $n=1$, there are two: $(1,0,0),(0,0,1)$. For $n=2$, there are four: $(0,1,0),(0,0,2),(1,0,1),(2,0,0)$. For $n \geq 3$ and odd, four: $\left(0, \frac{n-1}{2}, 1\right),\left(1, \frac{n-1}{2}, 0\right),\left(2, \frac{n-3}{2}, 1\right),\left(1, \frac{n-3}{2}, 2\right)$. For $n \geq 4$ and even, five: $\left(0, \frac{n}{2}, 0\right),\left(1, \frac{n-2}{2}, 1\right),\left(2, \frac{n-2}{2}, 0\right),\left(0, \frac{n-2}{2}, 2\right),\left(2, \frac{n-4}{2}, 2\right)$.

Corollary 4.8. The Gelfand-Kirillov dimension of $U\left(A^{\omega}\right)$ is 1 .

Proof. We have

$$
G K \operatorname{dim} U\left(A^{\omega}\right)=\limsup _{n \rightarrow \infty} \log _{n} \operatorname{dim} V^{(n)}=\lim _{n \rightarrow \infty} \frac{\ln \operatorname{dim} V^{(n)}}{\ln n}=1,
$$

since Corollary 4.7 implies that $\operatorname{dim} V^{(n)}$ is a polynomial of degree 1 .

Corollary 4.9. A $\mathbb{Z}$-grading of $U\left(A^{\omega}\right)$ is given by

$$
U\left(A^{\omega}\right)=U\left(A^{\omega}\right)_{-2} \oplus U\left(A^{\omega}\right)_{-1} \oplus U\left(A^{\omega}\right)_{0} \oplus U\left(A^{\omega}\right)_{1} \oplus U\left(A^{\omega}\right)_{2},
$$

where $U\left(A^{\omega}\right)_{n}=\operatorname{span}\left\{a^{i}(b a)^{j} b^{k} \mid j \geq 0,0 \leq i, k \leq 2, i-k=n\right\}$. 
Proof. Similar to [3, Proposition 3.5].

Our next goal is to compute the structure constants of $U\left(A^{\omega}\right)$.

Definition 4.10. For $j, \ell, r, m \geq 0$, we define the following polynomials:

$$
L_{j, \ell, r}^{m}=\sum_{t=0}^{j}(-1)^{j+t}\left(\begin{array}{l}
j \\
t
\end{array}\right) a^{\ell}(b a)^{j+m-t} b^{r} .
$$

Lemma 4.11. We have

$$
-L_{m+1, \ell, r}^{j-1}+L_{m, \ell, r}^{j-1}=L_{m, \ell, r}^{j}(j>0), \quad-L_{j, \ell, r}^{m+1}+L_{j, \ell, r}^{m}=L_{j+1, \ell, r}^{m} .
$$

Proof. Use Pascal's formula for binomial coefficients.

Lemma 4.12. If $j, m \geq 0$, then in $U\left(A^{\omega}\right)$ we have

$$
\begin{aligned}
(b a)^{j} \cdot a(b a)^{m} & =-\widehat{\delta}_{j, 0} L_{m, 2,1}^{j-1}+L_{j, 1,0}^{m}, \\
(b a)^{j} b \cdot(b a)^{m} & =-\widehat{\delta}_{m, 0} L_{j, 1,2}^{m-1}+L_{m, 0,1}^{j}, \\
(b a)^{j} b^{2} \cdot a^{2} & =-L_{j+1,1,1}^{0}+(b a)^{j+1}-(b a)^{j+2}+a^{2}(b a)^{j} b^{2} .
\end{aligned}
$$

Proof. For (10), we use induction on $j$. Clearly the claim is true for $j=0$. To prove it for $j=1$, we use induction on $m$. For $m=0$, Lemma 4.5 implies

$$
(b a) a=b a^{2}=-a b a-a^{2} b+a=-L_{0,2,1}^{0}+L_{1,1,0}^{0} .
$$

By the inductive hypothesis, we have

$$
\begin{aligned}
(b a) a(b a)^{m} & =(b a) a(b a)^{m-1} b a=\left(-L_{m-1,2,1}^{0}+L_{1,1,0}^{m-1}\right) b a \\
& =(-1)^{m} \sum_{t=0}^{m-1}(-1)^{t}\left(\begin{array}{c}
m-1 \\
t
\end{array}\right) a^{2}(b a)^{m-t-1} b^{2} a-a(b a)^{m+1}+a(b a)^{m} .
\end{aligned}
$$

Use the second relation of Lemma 4.5

$$
\begin{aligned}
(b a) a(b a)^{m}= & (-1)^{m} \sum_{t=0}^{m-1}(-1)^{t+1}\left(\begin{array}{c}
m-1 \\
t
\end{array}\right) a^{2}(b a)^{m-t} b \\
& +(-1)^{m} \sum_{t=0}^{m-1}(-1)^{t+1}\left(\begin{array}{c}
m-1 \\
t
\end{array}\right) a^{2}(b a)^{m-t-1} a b^{2} \\
& +(-1)^{m} \sum_{t=0}^{m-1}(-1)^{t}\left(\begin{array}{c}
m-1 \\
t
\end{array}\right) a^{2}(b a)^{m-t-1} b-a(b a)^{m+1}+a(b a)^{m} .
\end{aligned}
$$

Use Pascal's formula in the first sum and change index in the third sum:

$$
\begin{aligned}
(b a) a(b a)^{m}= & (-1)^{m+1}\left(a^{2}(b a)^{m} b+\sum_{t=1}^{m-1}(-1)^{t}\left(\begin{array}{c}
m \\
t
\end{array}\right) a^{2}(b a)^{m-t} b\right) \\
& +(-1)^{m} \sum_{t=1}^{m-1}(-1)^{t}\left(\begin{array}{c}
m-1 \\
t-1
\end{array}\right) a^{2}(b a)^{m-t} b \\
& +(-1)^{m} \sum_{t=0}^{m-1}(-1)^{t+1}\left(\begin{array}{c}
m-1 \\
t
\end{array}\right) a^{2}(b a)^{m-t-1} a b^{2} \\
& +(-1)^{m} \sum_{t=1}^{m}(-1)^{t-1}\left(\begin{array}{c}
m-1 \\
t-1
\end{array}\right) a^{2}(b a)^{m-t} b-a(b a)^{m+1}+a(b a)^{m}
\end{aligned}
$$


The first $m-1$ terms of the second and fourth sums cancel:

$$
\text { (ba) } a(b a)^{m}=-L_{m, 2,1}^{0}+(-1)^{m} \sum_{t=0}^{m-1}(-1)^{t+1}\left(\begin{array}{c}
m-1 \\
t
\end{array}\right) a^{2}(b a)^{m-t-1} a b^{2}+L_{1,1,0}^{m} .
$$

To complete the proof for $j=1$, it suffices to show that the second sum is 0 . But this holds since $a^{2}(b a)^{\ell} a=0$ for $\ell \geq 0$. To show this, we use induction on $\ell$; the claim is true for $\ell=0$ by the first equation of Lemma 4.5. For $\ell=1$, Lemma 4.5. implies $a^{2}(b a) a=a^{2}\left(-a b a-a^{2} b+a\right)=0$. For $\ell \geq 1$, Lemma 4.5 and the inductive hypothesis give $a^{2}(b a)^{\ell} a=a^{2}(b a)^{\ell-1}\left(-a b a-a^{2} b+a\right)=0$. We now consider the case $j \geq 1$. Using the inductive hypothesis and Lemma 4.5, we obtain

$$
\begin{aligned}
& (b a)^{j+1} a(b a)^{m}=b a(b a)^{j} a(b a)^{m}=b a\left(-L_{m, 2,1}^{j-1}+L_{j, 1,0}^{m}\right) \\
& =(-1)^{j} \sum_{t=0}^{j}(-1)^{t}\left(\begin{array}{l}
j \\
t
\end{array}\right)(b a) a(b a)^{j+m-t} \\
& =(-1)^{m+1} \sum_{t=0}^{j} \sum_{s=0}^{j+m-t}(-1)^{s}\left(\begin{array}{l}
j \\
t
\end{array}\right)\left(\begin{array}{c}
j+m-t \\
s
\end{array}\right) a^{2}(b a)^{j+m-t-s} b \\
& \quad+(-1)^{j+1} \sum_{t=0}^{j}(-1)^{t}\left(\begin{array}{l}
j \\
t
\end{array}\right) a(b a)^{j+m-t+1}+(-1)^{j} \sum_{t=0}^{j}(-1)^{t}\left(\begin{array}{l}
j \\
t
\end{array}\right) a(b a)^{j+m-t} .
\end{aligned}
$$

Use Pascal's formula in the second sum and change index in the third sum:

$$
\begin{aligned}
(b a)^{j+1} a(b a)^{m}= & (-1)^{m+1} \sum_{t=0}^{j} \sum_{s=0}^{j+m-t}(-1)^{s}\left(\begin{array}{l}
j \\
t
\end{array}\right)\left(\begin{array}{c}
j+m-t \\
s
\end{array}\right) a^{2}(b a)^{j+m-t-s} b \\
& +(-1)^{j+1} \sum_{t=0}^{j}(-1)^{t}\left(\begin{array}{c}
j+1 \\
t
\end{array}\right) a(b a)^{j+m-t+1} \\
& -(-1)^{j+1} \sum_{t=1}^{j}(-1)^{t}\left(\begin{array}{c}
j \\
t-1
\end{array}\right) a(b a)^{j+m-t+1} \\
& -(-1)^{j} \sum_{t=1}^{j}(-1)^{t}\left(\begin{array}{c}
j \\
t-1
\end{array}\right) a(b a)^{j+m-t+1}+a(b a)^{m} .
\end{aligned}
$$

The last two sums cancel and the previous expression simplifies to

$$
(-1)^{m+1} \sum_{t=0}^{j} \sum_{s=0}^{j+m-t}(-1)^{s}\left(\begin{array}{l}
j \\
t
\end{array}\right)\left(\begin{array}{c}
j+m-t \\
s
\end{array}\right) a^{2}(b a)^{j+m-t-s} b+L_{j+1,1,0}^{m} .
$$

To complete the proof, it suffices to show that

$$
\sum_{t=0}^{j} \sum_{s=0}^{j+m-t}(-1)^{s}\left(\begin{array}{l}
j \\
t
\end{array}\right)\left(\begin{array}{c}
j+m-t \\
s
\end{array}\right) a^{2}(b a)^{j+m-t-s} b=\sum_{t=0}^{m}(-1)^{t}\left(\begin{array}{c}
m \\
t
\end{array}\right) a^{2}(b a)^{j+m-t} b .
$$

Let $C_{r}$ denote the coefficient of $a^{2}(b a)^{j+m-r} b$ in the left side:

$$
\begin{aligned}
C_{r} & =\sum_{t=0}^{j} \sum_{s=0}^{j+m-t} \delta_{s+t, r}(-1)^{s}\left(\begin{array}{l}
j \\
t
\end{array}\right)\left(\begin{array}{c}
j+m-t \\
s
\end{array}\right)=\sum_{t=0}^{j}\left(\begin{array}{l}
j \\
t
\end{array}\right)(-1)^{r-t}\left(\begin{array}{c}
j+m-t \\
r-t
\end{array}\right) \\
& =(-1)^{r} \sum_{t=0}^{j}(-1)^{t}\left(\begin{array}{l}
j \\
t
\end{array}\right)\left(\begin{array}{c}
j+m-t \\
r-t
\end{array}\right)=(-1)^{r}\left(\begin{array}{c}
m \\
r
\end{array}\right) .
\end{aligned}
$$


For the last equality, see [22, Example 10.3]. This completes the proof of (1). The proof of (2) is obvious by using the anti-automorphism $\zeta$ :

$$
(b a)^{j} b(b a)^{m}=\zeta\left((b a)^{m} a(b a)^{j}\right)=-\widehat{\delta}_{m, 0} L_{j, 1,2}^{m-1}+L_{m, 0,1}^{j} .
$$

For (3), we use Lemma 4.5 and get

$$
(b a)^{j} b^{2} a^{2}=(b a)^{j}\left(-b a b-a b^{2}+b\right) a=-(b a)^{j+2}-(b a)^{j} a b^{2} a+(b a)^{j+1} .
$$

Write $T=-(b a)^{j} a b^{2} a$. Lemma 4.5 implies

$$
T=-(b a)^{j} a\left(-b a b-a b^{2}+b\right)=(b a)^{j} a(b a) b+\delta_{j, 0} a^{2} b^{2}-(b a)^{j} a b .
$$

Using (10) and Lemma 4.11 we get

$$
\begin{aligned}
T & =\left(-\widehat{\delta}_{j, 0} L_{1,2,1}^{j-1}+L_{j, 1,0}^{1}+\widehat{\delta}_{j, 0} L_{0,2,1}^{j-1}-L_{j, 1,0}^{0}\right) b+\delta_{j, 0} a^{2} b^{2} \\
& =\widehat{\delta}_{j, 0} L_{0,2,1}^{j} b-L_{j+1,1,0}^{0} b+\delta_{j, 0} a^{2} b^{2}=a^{2}(b a)^{j} b^{2}-L_{j+1,1,1}^{0} .
\end{aligned}
$$

Using $T$ in (4) completes the proof of (3).

Theorem 4.13. The structure constants of $U\left(A^{\omega}\right)$ are

(5) $a^{i}(b a)^{j} b^{k} \cdot a^{\ell}(b a)^{m} b^{n}=a^{i}(b a)^{j+k+\ell+m} b^{n}$, if $(k, \ell)=(0,0)$ or $(k, \ell)=(1,1)$,

$$
\begin{aligned}
a^{i}(b a)^{j} \cdot a(b a)^{m} b^{n} & =-\delta_{i, 0} \widehat{\delta}_{n, 2} \widehat{\delta}_{j, 0} L_{m, 2, n+1}^{j-1}+\widehat{\delta}_{i, 2} L_{j, i+1, n}^{m}, \\
a^{i}(b a)^{j} b \cdot(b a)^{m} b^{n} & =-\delta_{n, 0} \widehat{\delta}_{i, 2} \widehat{\delta}_{m, 0} L_{j, i+1,2}^{m-1}+\widehat{\delta}_{n, 2} L_{m, i, n+1}^{j}, \\
a^{i}(b a)^{j} b^{2} \cdot a(b a)^{m} b^{n} & =-\delta_{n, 0} \widehat{\delta}_{i, 2} L_{j, i+1,2}^{m}+\widehat{\delta}_{n, 2} L_{m+1, i, n+1}^{j}, \\
a^{i}(b a)^{j} b \cdot a^{2}(b a)^{m} b^{n} & =-\delta_{i, 0} \widehat{\delta}_{n, 2} L_{m, 2, n+1}^{j}+\widehat{\delta}_{i, 2} L_{j+1, i+1, n}^{m}, \\
a^{i}(b a)^{j} b^{2} \cdot(b a)^{m} b^{n} & =\delta_{m, 0} \delta_{n, 0} a^{i}(b a)^{j} b^{2} \\
a^{i}(b a)^{j} \cdot a^{2}(b a)^{m} b^{n} & =\delta_{i, 0} \delta_{j, 0} a^{2}(b a)^{m} b^{n}
\end{aligned}
$$

together with

$$
\begin{aligned}
& a^{i}(b a)^{j} b^{2} \cdot a^{2}(b a)^{m} b^{n} \\
& =\sum_{k=0}^{j+1}(-1)^{k+j}\left(\begin{array}{c}
j+1 \\
k
\end{array}\right)\left[-\delta_{n, 0} \delta_{i, 0} \widehat{\delta}_{m, 0} L_{j-k+1,2,2}^{m-1}+\widehat{\delta}_{n, 2} \widehat{\delta}_{i, 2} L_{m, i+1, n+1}^{j-k+1}\right] \\
& \quad+a^{i}(b a)^{j+m+1} b^{n}-a^{i}(b a)^{j+m+2} b^{n}+\delta_{i, 0} \delta_{m, 0} \delta_{n, 0} a^{2}(b a)^{j} b^{2} .
\end{aligned}
$$

Proof. For (5), use the associativity of $U\left(A^{\omega}\right)$. For (6) and (7) use Lemma 4.5 and equations (11) and (2) of Lemma 4.12, For (8), Lemma 4.5) implies

$$
\begin{aligned}
a^{i}(b a)^{j} b^{2} a(b a)^{m} b^{n} & =a^{i}(b a)^{j}\left(-b a b-a b^{2}+b\right)(b a)^{m} b^{n} \\
& =-a^{i}(b a)^{j+1} b(b a)^{m} b^{n}-\delta_{m, 0} \delta_{n, 0} a^{i}(b a)^{j} a b^{2}+a^{i}(b a)^{j} b(b a)^{m} b^{n} .
\end{aligned}
$$

Using (6) and (7) and Lemma 4.11 we obtain (8):

$$
\begin{aligned}
a^{i}(b a)^{j} b^{2} a(b a)^{m} b^{n}= & \delta_{n, 0} \widehat{\delta}_{i, 2} \widehat{\delta}_{m, 0} L_{j+1, i+1,2}^{m-1}-\widehat{\delta}_{n, 2} L_{m, i, n+1}^{j+1} \\
& -\delta_{m, 0} \delta_{n, 0} \widehat{\delta}_{i, 2} L_{j, i+1,2}^{0}-\delta_{n, 0} \widehat{\delta}_{i, 2} \widehat{\delta}_{m, 0} L_{j, i+1,2}^{m-1}+\widehat{\delta}_{n, 2} L_{m, i, n+1}^{j} \\
= & -\delta_{n, 0} \widehat{\delta}_{i, 2} \widehat{\delta}_{m, 0} L_{j, i+1,2}^{m}+\widehat{\delta}_{n, 2} L_{m+1, i, n+1}^{j}-\delta_{m, 0} \widehat{\delta}_{n, 0} \widehat{\delta}_{i, 2} L_{j, i+1,2}^{0} .
\end{aligned}
$$

For (9) use (8) and the anti-automorphism $\zeta$. The proofs of (10) and (11) are obvious by Lemma 4.5. For (12), we use (3) of Lemma 4.12 and obtain

$$
\begin{aligned}
a^{i}(b a)^{j} b^{2} a^{2}(b a)^{m} b^{n}= & -a^{i} L_{j+1,1,1}^{0}(b a)^{m} b^{n}+a^{i}(b a)^{j+1}(b a)^{m} b^{n} \\
& -a^{i}(b a)^{j+2}(b a)^{m} b^{n}+a^{i+2}(b a)^{j} b^{2}(b a)^{m} b^{n} .
\end{aligned}
$$


Using Lemma 4.5, we get

$$
\begin{aligned}
a^{i}(b a)^{j} b^{2} a^{2}(b a)^{m} b^{n}= & -\widehat{\delta}_{i, 2} L_{j+1, i+1,1}^{0}(b a)^{m} b^{n}+a^{i}(b a)^{j+1+m} b^{n} \\
& -a^{i}(b a)^{j+m+2} b^{n}+\delta_{i, 0} \delta_{m, 0} \delta_{n, 0} a^{2}(b a)^{j} b^{2} .
\end{aligned}
$$

Write $A=L_{j+1, i+1,1}^{0}(b a)^{m} b^{n}$ and use (77) to obtain

$$
\begin{aligned}
A & =\sum_{k=0}^{j+1}(-1)^{k+j+1}\left(\begin{array}{c}
j+1 \\
k
\end{array}\right) a^{i+1}(b a)^{j-k+1} b(b a)^{m} b^{n} \\
& =\sum_{k=0}^{j+1}(-1)^{k+j+1}\left(\begin{array}{c}
j+1 \\
k
\end{array}\right)\left[-\delta_{n, 0} \delta_{i, 0} \widehat{\delta}_{m, 0} L_{j-k+1,2,2}^{m-1}+\widehat{\delta}_{n, 2} \widehat{\delta}_{i, 2} L_{m, i+1, n+1}^{j-k+1}\right] .
\end{aligned}
$$

Using $A$ in (13) completes the proof of (12).

Our next goal is to describe the center $Z\left(U\left(A^{\omega}\right)\right)$ of $U\left(A^{\omega}\right)$.

Notation 4.14. We consider the following functions:

$$
\begin{array}{ccccc} 
& \gamma_{1}(m) & \gamma_{2}(m) & \gamma_{3}(m) & \gamma_{4}(m) \\
m \text { even } & m+1 & -3 & 0 & -m+2 \\
m \text { odd } & -(m-3) & -1 & -2 & m
\end{array}
$$

Definition 4.15. We consider the following elements:

$$
\begin{aligned}
\mathcal{Z}(m)= & \widehat{\delta}_{m 2} \sum_{j=1}^{m-2}(-1)^{j+1}\left(\begin{array}{c}
m-1 \\
j-1
\end{array}\right)\left((b a)^{j}-a(b a)^{j-1} b\right)+\gamma_{1}(m)(b a)^{m-1} \\
& +\gamma_{2}(m)(b a)^{m}+\gamma_{3}(m) a(b a)^{m-1} b+\gamma_{4}(m) a(b a)^{m-2} b+3 a^{2}(b a)^{m-2} b^{2} .
\end{aligned}
$$

Theorem 4.16. The center of $U\left(A^{\omega}\right)$ is the polynomial algebra in $\mathcal{Z}(m), m \geq 2$ :

$$
Z\left(U\left(A^{\omega}\right)\right)=\mathbb{F}[\mathcal{Z}(m) \mid m \geq 2] .
$$

Proof. By Corollary 4.9 we know $Z\left(U\left(A^{\omega}\right)\right)$ is graded. Thus if $z$ is central and $z=z_{-2}+z_{-1}+z_{0}+z_{1}+z_{2}$ is its decomposition into homogenous components, then each $z_{i}$ is itself central. We now show that $z \in U\left(A^{\omega}\right)_{0}$. First assume

$$
0 \neq z_{-2}=\sum_{j \geq 0} s_{j}(b a)^{j} b^{2} \in Z\left(U\left(A^{\omega}\right)\right), \quad s_{j} \in \mathbb{F} .
$$

It follows that

$$
0=z_{-2} a-a z_{-2}=\sum_{j \geq 0} s_{j}(b a)^{j} b^{2} a-\sum_{j \geq 0} s_{j} a(b a)^{j} b^{2} .
$$

Using (8) of Theorem 4.13, we see that this element is 0 :

$\sum_{j \geq 0} \sum_{t=0}^{j}(-1)^{j+t+1}\left(\begin{array}{l}j \\ t\end{array}\right) s_{j} a(b a)^{j-t} b^{2}-\sum_{j \geq 0} s_{j}(b a)^{j+1} b+\sum_{j \geq 0} s_{j}(b a)^{j} b-\sum_{j \geq 0} s_{j} a(b a)^{j} b^{2}$.

Comparing the coefficients on both sides gives $s_{j}=0$ for all $j$. Now assume

$$
0 \neq z_{2}=\sum_{j \geq 0} s_{j} a^{2}(b a)^{j} \in Z\left(U\left(A^{\omega}\right)\right), \quad s_{j} \in \mathbb{F} .
$$

It follows that

$$
0=b z_{2}-z_{2} b=\sum_{j \geq 0} s_{j} b a^{2}(b a)^{j}-\sum_{j \geq 0} s_{j} a^{2}(b a)^{j} b .
$$


Applying the anti-automorphism $\zeta$ to both sides gives

$$
\sum_{j \geq 0} s_{j}(b a)^{j} b^{2} a=\sum_{j \geq 0} s_{j} a(b a)^{j} b^{2} .
$$

Hence $\left[\sum_{j \geq 0} s_{j}(b a)^{j} b^{2}, a\right]=0$, contradicting the previous case. Next assume

$$
0 \neq z_{1}=\sum_{j \geq 0} s_{j} a(b a)^{j}+\sum_{\ell \geq 0} t_{\ell} a^{2}(b a)^{\ell} b \in Z\left(U\left(A^{\omega}\right)\right), \quad s_{j}, t_{\ell} \in \mathbb{F} .
$$

It follows that

$$
0=b z_{1}-z_{1} b=\sum_{j \geq 0} s_{j}(b a)^{j+1}+\sum_{\ell \geq 0} t_{\ell} b a^{2}(b a)^{\ell} b-\sum_{j \geq 0} s_{j} a(b a)^{j} b-\sum_{\ell \geq 0} t_{\ell} a^{2}(b a)^{\ell} b^{2} .
$$

Using (9) of Theorem 4.13 gives

$$
\begin{aligned}
0= & \sum_{j \geq 0} s_{j}(b a)^{j+1}-\sum_{\ell \geq 0} \sum_{t=0}^{\ell}(-1)^{\ell+t}\left(\begin{array}{l}
\ell \\
t
\end{array}\right) t_{\ell} a^{2}(b a)^{\ell-t} b^{2}-\sum_{\ell \geq 0} t_{\ell} a(b a)^{\ell+1} b \\
& +\sum_{\ell \geq 0} t_{\ell} a(b a)^{\ell} b-\sum_{j \geq 0} s_{j} a(b a)^{j} b-\sum_{\ell \geq 0} t_{\ell} a^{2}(b a)^{\ell} b^{2} .
\end{aligned}
$$

Comparing the coefficients on both sides gives $s_{j}=0=t_{\ell}$ for all $j, \ell$. Similarly we can show that $Z\left(U\left(A^{\omega}\right)\right) \cap U\left(A^{\omega}\right)_{-1}=0$. Therefore $z \in U\left(A^{\omega}\right)_{0}$. Now $U\left(A^{\omega}\right)_{0}$ is a commutative subalgebra: any element in $U\left(A^{\omega}\right)_{0}$ is a linear combination of $(b a)^{j}$, $a(b a)^{k} b, a^{2}(b a)^{\ell} b^{2}$ for $j, k, \ell \geq 0$, and these elements commute by Theorem 4.13 Using the anti-automorphism $\zeta$, we see that an element in $U\left(A^{\omega}\right)_{0}$ commutes with $a$ if and only if it commutes with $b$. So it suffices to determine the elements that commute with $a$. Without loss of generality, we choose

$$
z=\sum_{j=0}^{m} \sum_{i=0}^{2} s_{i, j} a^{i}(b a)^{j} b^{i} \in U\left(A^{\omega}\right)_{0}, \quad m \geq 0, s_{i, j} \in \mathbb{F} .
$$

By the relations of Lemma 4.5 we have

$$
a z=\sum_{j=0}^{m} s_{0, j} a(b a)^{j}+\sum_{j=0}^{m} s_{1, j} a^{2}(b a)^{j} b .
$$

On the other hand,

$$
z a=\sum_{j=0}^{m} s_{0, j}(b a)^{j} a+\sum_{j=0}^{m} s_{1, j} a(b a)^{j+1}+\sum_{j=0}^{m} s_{2, j} a^{2}(b a)^{j} b^{2} a .
$$

Using (6) and (8) of Theorem 4.13 we obtain

$$
\begin{aligned}
z a= & s_{0,0} a+\sum_{j=1}^{m} s_{0, j}\left(-a^{2}(b a)^{j-1} b+\sum_{t=0}^{j}(-1)^{j+t}\left(\begin{array}{l}
j \\
t
\end{array}\right) a(b a)^{j-t}\right) \\
& +\sum_{j=0}^{m} s_{1, j} a(b a)^{j+1}-\sum_{j=0}^{m} s_{2, j} a^{2}(b a)^{j+1} b+\sum_{j=0}^{m} s_{2, j} a^{2}(b a)^{j} b .
\end{aligned}
$$

We write

$$
A=\sum_{j=1}^{m} \sum_{t=0}^{j}(-1)^{j+t}\left(\begin{array}{l}
j \\
t
\end{array}\right) s_{0, j} a(b a)^{j-t}, \quad E=\sum_{j=0}^{m} s_{1, j} a(b a)^{j+1} .
$$


We obtain

$$
\begin{aligned}
& A+E=\sum_{r=0}^{m}\left(\sum_{j=1}^{m}(-1)^{r}\left(\begin{array}{l}
j \\
r
\end{array}\right) s_{0, j}\right) a(b a)^{r}+\sum_{j=1}^{m+1} s_{1, j-1} a(b a)^{j} \\
& =\sum_{j=1}^{m} s_{0, j} a+\sum_{r=1}^{m}\left(\sum_{j=1}^{m}(-1)^{r}\left(\begin{array}{l}
j \\
r
\end{array}\right) s_{0, j}+s_{1, r-1}\right) a(b a)^{r}+s_{1, m} a(b a)^{m+1} .
\end{aligned}
$$

Using (16) in (15) gives

$$
\begin{aligned}
z a= & \left(s_{0,0}+\sum_{j=1}^{m} s_{0, j}\right) a-\sum_{j=1}^{m} s_{0, j} a^{2}(b a)^{j-1} b \\
& +\sum_{r=1}^{m}\left(\sum_{j=1}^{m} s_{0, j}(-1)^{r}\left(\begin{array}{l}
j \\
r
\end{array}\right)+s_{1, r-1}\right) a(b a)^{r}+s_{1, m} a(b a)^{m+1} \\
& -\sum_{j=0}^{m} s_{2, j} a^{2}(b a)^{j+1} b+\sum_{j=0}^{m} s_{2, j} a^{2}(b a)^{j} b .
\end{aligned}
$$

Changing index in the second and fourth sums and combining coefficients gives

$$
\begin{aligned}
z a= & \left(s_{0,0}+\sum_{j=1}^{m} s_{0, j}\right) a+\left(-s_{0,1}+s_{2,0}\right) a^{2} b+\sum_{j=1}^{m-1}\left(-s_{0, j+1}-s_{2, j-1}+s_{2, j}\right) a^{2}(b a)^{j} b \\
& +\sum_{r=1}^{m}\left(\sum_{j=1}^{m} s_{0, j}(-1)^{r}\left(\begin{array}{l}
j \\
r
\end{array}\right)+s_{1, r-1}\right) a(b a)^{r}+s_{1, m} a(b a)^{m+1} \\
& +\left(-s_{2, m-1}+s_{2, m}\right) a^{2}(b a)^{m} b-s_{2, m} a^{2}(b a)^{m+1} b .
\end{aligned}
$$

Comparing the coefficients in this expression with (14), we get this linear system:

$$
\left\{\begin{array}{l}
\sum_{j=1}^{m} s_{0, j}=0, \\
-s_{0,1}+s_{2,0}-s_{1,0}=0, \\
-s_{0, j+1}-s_{2, j-1}+s_{2, j}-s_{1, j}=0 \quad(1 \leq j \leq m-1), \\
(-1)^{r} \sum_{j=1}^{m}\left(\begin{array}{c}
j \\
r
\end{array}\right) s_{0, j}+s_{1, r-1}-s_{0, r}=0 \quad(1 \leq r \leq m), \\
s_{2, m}=s_{1, m}=s_{2, m-1}=0 .
\end{array}\right.
$$

For $m<2$, the only solution is trivial. For $m \geq 2$, a calculation (details omitted) shows that $(\mathcal{T})$ has $m-1$ linearly independent solutions. For each $m$, we have the following solution:

$$
\begin{aligned}
& s_{2, m-2}=1, \quad s_{2, j}=0(j \neq m-2), \quad s_{0, j}=\frac{1}{3}(-1)^{j+1}\left(\begin{array}{c}
m-1 \\
j-1
\end{array}\right)(1 \leq j \leq m-2), \\
& s_{0, m-1}=\left\{\begin{array}{ll}
(m+1) / 3 & \text { if } m \text { is even } \\
(3-m) / 3 & \text { if } m \text { is odd }
\end{array}, \quad s_{0, m}=\left\{\begin{array}{ll}
-1 & \text { if } m \text { is even } \\
-1 / 3 & \text { if } m \text { is odd }
\end{array},\right.\right. \\
& s_{1, i-1}=-s_{0, i}(1 \leq i \leq m-2), \quad s_{1, m-2}=-s_{0, m-1}+1 \text {, } \\
& s_{1, m-1}=\left\{\begin{array}{ll}
0 & \text { if } m \text { is even } \\
-2 / 3 & \text { if } m \text { is odd }
\end{array}, \quad s_{1, m}=0 .\right.
\end{aligned}
$$

Using this solution for $z$, and observing that any solution for $m-1$ is also a solution for $m$, we obtain a complete list of linearly independent solutions for (T). 
4.2. The alternating sum. The structure constants for $A^{\omega}$ are 0 , the set of ideal generators is empty, and hence $U\left(A^{\omega}\right)$ is the free associative algebra on $a$ and $b$. The Gelfand-Kirillov dimension of $U\left(A^{\omega}\right)$ is $\infty$.

4.3. The cyclic sum. The results are identical to those for the symmetric sum, since the structure constants are

$$
\left[e_{2}, e_{1}, e_{1}\right]=e_{1}, \quad\left[e_{1}, e_{2}, e_{2}\right]=e_{2}, \quad\left[e_{1}, e_{1}, e_{1}\right]=\left[e_{2}, e_{2}, e_{2}\right]=0 .
$$

4.4. The Lie family, $q=\infty$. The structure constants for $A^{\omega_{L}^{\infty}}$ are determined by

$$
\left[e_{1}, e_{2}, e_{1}\right]=2 e_{1}, \quad\left[e_{2}, e_{2}, e_{1}\right]=-2 e_{2} .
$$

Lemma 4.17. The universal associative envelope $U\left(A^{\omega_{L}^{\infty}}\right)$ is isomorphic to the down-up algebra $A(2,-1,-2)$.

Proof. We have $U\left(A^{\omega_{L}^{\infty}}\right)=F\langle a, b\rangle / I$, where $I$ is the ideal generated by these two elements, which form a Gröbner basis: $b^{2} a-2 b a b+a b^{2}+2 b, b a^{2}-2 a b a+a^{2} b+2 a$.

Remark 4.18. If we replace $\omega_{L}^{\infty}$ by $\omega^{\prime}=[[-,-],-]$ then we get the 2 -dimensional simple Lie triple system $A^{\omega^{\prime}}$ with relations $\left[e_{1}, e_{2}, e_{1}\right]=2 e_{1},\left[e_{1}, e_{2}, e_{2}\right]=-2 e_{2}$; the results for $U\left(A^{\omega^{\prime}}\right)$ are identical to those for $\omega_{L}^{\infty}$.

Benkart and Roby [3] showed that the down-up algebra $A(2,-1,-2)$ is isomorphic to the universal associative envelope $U\left(\mathfrak{s l}_{2}\right)$ of the simple Lie algebra of $2 \times 2$ matrices of trace 0 with basis $\{h, e, f\}$ and relations $[e, f]=h,[h, e]=2 e$, and $[h, f]=-2 f$. In $U\left(\mathfrak{s l}_{2}\right)$ we have $e f-f e=h, h e-e h=2 e, h f-f h=-2 f$.

Lemma 4.19. If $\ell, k, m, j \geq 0$ then in $U\left(\mathfrak{s l}_{2}\right)$ we have

$$
\begin{aligned}
e^{\ell} \cdot h^{k} & =\sum_{q=0}^{k}(-1)^{q} 2^{q}\left(\begin{array}{l}
k \\
q
\end{array}\right) \ell^{q} h^{k-q} e^{\ell}, \\
h^{k} \cdot f^{m} & =\sum_{q=0}^{k}(-1)^{q} 2^{q}\left(\begin{array}{l}
k \\
q
\end{array}\right) m^{q} f^{m} h^{k-q}, \\
e^{\ell} \cdot f^{j} & =\ell ! j ! \sum_{r=0}^{\min (j, \ell)} \frac{f^{j-r}}{(j-r) !}\left(\begin{array}{c}
h-j-\ell+2 r \\
r
\end{array}\right) \frac{e^{\ell-r}}{(\ell-r) !} .
\end{aligned}
$$

Proof. For (17), we use induction on $k$. The claim is clear for $k=0$. To prove the claim for $k=1$, we use induction on $\ell$. For $\ell=1$, the claim holds since $e h=h e-2 e$. Assume that $\ell \geq 1$. By the inductive hypothesis we have

$$
e^{\ell+1} h=e e^{\ell} h=\left(e h e^{\ell}-2 \ell e^{\ell+1}\right)=h e^{\ell+1}-2 e^{\ell+1}-2 \ell e^{\ell+1},
$$

so the claim is true for $k=1$. For $k \geq 1$, the inductive hypothesis implies

$$
\begin{aligned}
& e^{\ell} h^{k+1}=\sum_{q=0}^{k}(-1)^{q} 2^{q}\left(\begin{array}{l}
k \\
q
\end{array}\right) \ell^{q} h^{k-q} e^{\ell} h=\sum_{q=0}^{k}(-1)^{q} 2^{q}\left(\begin{array}{l}
k \\
q
\end{array}\right) \ell^{q} h^{k-q}\left(h e^{\ell}-2 \ell e^{\ell}\right) \\
& =\sum_{q=0}^{k}(-1)^{q} 2^{q}\left(\begin{array}{l}
k \\
q
\end{array}\right) \ell^{q} h^{k-q+1} e^{\ell}+\sum_{q=1}^{k+1}(-1)^{q} 2^{q}\left(\begin{array}{c}
k \\
q-1
\end{array}\right) \ell^{q} h^{k-q+1} e^{\ell} \\
& =h^{k+1} e^{\ell}+\sum_{q=1}^{k}(-1)^{q} 2^{q}\left[\left(\begin{array}{c}
k \\
q
\end{array}\right)+\left(\begin{array}{c}
k \\
q-1
\end{array}\right)\right] \ell^{q} h^{k+1-q} e^{\ell}+(-1)^{k+1} 2^{k+1} \ell^{k+1} e^{\ell} .
\end{aligned}
$$


Using Pascal's formula for binomial coefficients we obtain

$$
e^{\ell} h^{k+1}=h^{k+1} e^{\ell}+\sum_{q=1}^{k}(-1)^{q} 2^{q}\left(\begin{array}{c}
k+1 \\
q
\end{array}\right) \ell^{q} h^{k+1-q} e^{\ell}+(-1)^{k+1} 2^{k+1} \ell^{k+1} e^{\ell} .
$$

This proves (17), and (18) is similar; for (19), see Humphreys [14, Lemma 26.2].

Theorem 4.20. The structure constants of $U\left(\mathfrak{s l}_{2}\right)$ are

$$
\begin{aligned}
\left(f^{i} h^{j} e^{k}\right) \cdot\left(f^{\ell} h^{m} e^{n}\right)= & k ! \ell ! \sum_{r=0}^{\min (\ell, k)} \sum_{q=0}^{j} \sum_{i=0}^{m}(-1)^{q+i} 2^{q+i}\left(\begin{array}{c}
j \\
q
\end{array}\right)\left(\begin{array}{c}
m \\
i
\end{array}\right) \frac{(\ell-r)^{q}}{(\ell-r) !} \frac{(k-r)^{i}}{(k-r) !} \\
& \times f^{\ell-r+i} h^{j-q}\left(\begin{array}{c}
h-k-\ell+2 r \\
r
\end{array}\right) h^{m-i} e^{k-r+n} .
\end{aligned}
$$

Remark 4.21. Using Theorem 4.20 and the homomorphism $\psi: \mathfrak{s l}_{2} \rightarrow A(2,-1,1)$ from [3], we obtain the structure constants of $U\left(A^{\omega_{L}^{\infty}}\right)$ with respect to the basis $\mathfrak{B}_{2}$ with $\left(c_{1}, c_{2}\right)=(-1,0)$ (see Lemma 4.3).

4.5. The Lie family, $q=\frac{1}{2}$. The structure constants for $A^{\omega_{L}^{1 / 2}}$ are zero.

Lemma 4.22. The universal associative envelope $U\left(A^{\omega_{L}^{1 / 2}}\right)$ is isomorphic to the down-up algebra $A(0,1,0)$.

Proof. We have $U\left(A_{L}^{\omega_{L}^{1 / 2}}\right)=F\langle a, b\rangle / I$, where $I$ is the ideal generated by these two elements, which form a Gröbner basis: $b^{2} a-a b^{2}, b a^{2}-a^{2} b$.

Remark 4.23. If we replace $\omega_{L}^{1 / 2}$ by $\omega^{\prime \prime}=\left[--_{-},-\right]$then we get an anti-Lie triple system $A^{\omega^{\prime \prime}}$, and the results for $U\left(A^{\omega^{\prime \prime}}\right)$ are the same as those for $A^{\omega_{L}^{1 / 2}}$.

Lemma 4.24. If $i, j \geq 0$, then in $U\left(A^{\omega_{L}^{1 / 2}}\right)$ we have

$$
\begin{aligned}
b^{i} \cdot a^{j} & = \begin{cases}a^{j-1}(b a) b^{i-1} & \text { if } i, j \text { are both odd, } \\
a^{j} b^{i} & \text { otherwise. }\end{cases} \\
(b a)^{j} \cdot a^{i} & = \begin{cases}a^{i+1}(b a)^{j-1} b & \text { if } i \text { is odd }, j \neq 0, \\
a^{i}(b a)^{j} & \text { otherwise. }\end{cases} \\
b^{i} \cdot(b a)^{j} & = \begin{cases}a(b a)^{j-1} b^{i+1} & \text { if } i \text { is odd, } j \neq 0, \\
(b a)^{j} b^{i} & \text { otherwise. }\end{cases} \\
(b a)^{i} \cdot a(b a)^{j} & = \begin{cases}a^{2 j+2}(b a)^{i-j-1} b^{2 j+1} & \text { if } i>j, \\
a^{2 i+1}(b a)^{j-i} b^{2 i} & \text { otherwise. }\end{cases}
\end{aligned}
$$

Proof. For (20), we use induction on $i$. The claim is clear for $i=0$. To prove the claim for $i=1$ we use induction on $j$. For $j=0$ or 1 , the claim is obvious. For $j=2$ the claim holds since $b a^{2}=a^{2} b$. We now prove the claim for $j \geq 2$. By the inductive hypothesis, we have

$b a^{j}=\left\{\begin{array}{ll}a^{j-2} b a a & \text { if } j-1 \text { is odd } \\ a^{j-1} b a & \text { otherwise }\end{array}=\left\{\begin{array}{ll}a^{j-1} b a & \text { if } j \text { is odd } \\ a^{j-2} b a a & \text { otherwise }\end{array}=\left\{\begin{array}{ll}a^{j-1} b a & \text { if } j \text { is odd } \\ a^{j} b & \text { otherwise }\end{array}\right.\right.\right.$. 
So the claim holds for $i=1$. We now consider the case $i \geq 1$. If $i+1$ is odd, then the inductive hypothesis implies

$$
b^{i+1} a^{j}=b b^{i} a^{j}=b a^{j} b^{i}=\left\{\begin{array}{ll}
a^{j-1}(b a) b^{i} & \text { if } j \text { is odd } \\
a^{j} b^{i+1} & \text { otherwise }
\end{array} .\right.
$$

If $i+1$ is even, then the inductive hypothesis gives

$$
b^{i+1} a^{j}=b b^{i} a^{j}=\left\{\begin{array}{ll}
b a^{j-1}(b a) b^{i-1} & \text { if } j \text { is odd } \\
b a^{j} b^{i} & \text { otherwise }
\end{array}=\left\{\begin{array}{ll}
a^{j-1} b(b a) b^{i-1} & \text { if } j \text { is odd } \\
a^{j} b^{i+1} & \text { otherwise }
\end{array} .\right.\right.
$$

Using $b^{2} a=a b^{2}$ we get $b^{i+1} a^{j}=a^{j-1} a b^{2} b^{i-1}$ if $j$ is odd, $a^{j} b^{i+1}$ otherwise; in both cases the result is $a^{j} b^{i+1}$. This completes the proof of (20).

For (21) we use induction on $i$. The claim is obvious for $i=0$. To prove the claim for $i=1$, we use induction on $j$. For $j=0$, the claim is obvious. For $j=1$, the claim holds by using $b a^{2}=a^{2} b$. We now consider the case of general $j$. By the inductive hypothesis, we have $(b a)^{j} a=b a(b a)^{j-1} a=b a a^{2}(b a)^{j-2} b=$ $a^{2} b a(b a)^{j-2}=a^{2}(b a)^{j-1} b$. So the claim is true for $i=1$. We now consider the case $i \geq 1$. The claim is obvious for $j=0$, so we assume that $j \neq 0$. If $i+1$ is odd, then the inductive hypothesis implies $(b a)^{j} a^{i+1}=a^{i}(b a)^{j} a=a^{i+2}(b a)^{j-1} b$. If $i+1$ is even, then $(b a)^{j} a^{i+1}=a^{i+1}(b a)^{j-1} b a=a^{i+1}(b a)^{j}$. This completes the proof of (21). The proof of (22) follows by using the anti-automorphism $\eta$ from [3] of the down-up algebra $A(0,1,0)$.

For (23), we use induction on $i$. The claim is obvious for $i=0$. To prove the claim for $i=1$, we use induction on $j$. The claim holds for $j=0$ by using $(b a) a=a^{2} b$. For $j \geq 1, b a^{2}=a^{2} b$ and (22) imply $b a a(b a)^{j}=a^{2} b(b a)^{j}=a^{3}(b a)^{j-1} b^{2}$. So the claim is true for $i=1$. We now consider the case of $i>1$. By the inductive hypothesis, we have

$$
(b a)^{i+1} a(b a)^{j}=b a(b a)^{i} a(b a)^{j}=\left\{\begin{array}{ll}
b a a^{2 j+2}(b a)^{i-j-1} b^{2 j+1} & \text { if } i>j \\
b a a^{2 i+1}(b a)^{j-i} b^{2 i} & \text { if } i \leq j
\end{array} .\right.
$$

Therefore

$$
(b a)^{i+1} a(b a)^{j}=\left\{\begin{array}{ll}
b a^{2 j+3}(b a)^{i-j-1} b^{2 j+1} & \text { if } i>j \\
b a^{2 i+2}(b a)^{j-i} b^{2 i} & \text { if } i \leq j
\end{array} .\right.
$$

Two cases need to be considered. (I) If $i+1>j$, then $i=j$ or $i>j$. Hence,

$$
(b a)^{i+1} a(b a)^{j}=\left\{\begin{array}{ll}
b a^{2 j+3}(b a)^{i-j-1} b^{2 j+1} & \text { if } i>j \\
b a^{2 i+2} b^{2 i} & \text { if } i=j
\end{array} .\right.
$$

Using (20) we obtain

$$
(b a)^{i+1} a(b a)^{j}=\left\{\begin{array}{ll}
a^{2 j} b a^{3}(b a)^{i-j-1} b^{2 j+1}=a^{2 j+2}(b a)^{i-j} b^{2 j+1} & \text { if } i>j \\
a^{2 i+2} b^{2 i+1} & \text { if } i=j
\end{array} .\right.
$$

Therefore, $(b a)^{i+1} a(b a)^{j}=a^{2 j+2}(b a)^{i-j} b^{2 j+1}$. (II) If $i+1 \leq j$, then $i<j$. Hence $(b a)^{i+1} a(b a)^{j}=b a^{2 i+2}(b a)^{j-i} b^{2 i}$. Using (20) and (22) we obtain $(b a)^{i+1} a(b a)^{j}=$ $a^{2 i+2} b(b a)^{j-i} b^{2 i}=a^{2 i+3}(b a)^{j-i-1} b^{2(i+1)}$. Combining the results of (I) and (II) completes the proof of (23). 
Theorem 4.25. The structure constants of $U\left(A^{\omega_{L}^{1 / 2}}\right)$ are

$$
\begin{aligned}
& a^{i}(b a)^{j} b^{k} \cdot a^{\ell}(b a)^{m} b^{n} \\
& \quad= \begin{cases}a^{i+\ell-1}(b a)^{j+m+1} b^{k-1+n} & \text { if } k, \ell \text { are both odd, } \\
a^{i+\ell}(b a)^{j+m} b^{k+n} & \text { if } k, \ell \text { are both even, } \\
\chi_{j, m} a^{2 m+i+\ell+1}(b a)^{j-m-1} b^{2 m+k+n+1} & \\
\quad+\left(1-\chi_{j, m}\right) a^{2 j+i+\ell}(b a)^{m-j} b^{2 j+k+n} & \text { if } k \text { is even, } \ell \text { is odd, } \\
\chi_{j, m-1} a^{2 m+i+\ell}(b a)^{j-m} b^{2 m+k+n} & \\
\quad+\left(1-\chi_{j, m-1}\right) a^{2 j+i+\ell+1}(b a)^{m-j-1} b^{2 j+k+n+1} & \text { if } k \text { is odd, } \ell \text { is even, }\end{cases}
\end{aligned}
$$

where $\chi_{\ell, t}=1$ if $\ell>t$ and 0 otherwise.

Proof. We use equations (20), (21) and (22). If $k$ and $\ell$ are odd, then

$$
a^{i}(b a)^{j} b^{k} \cdot a^{\ell}(b a)^{m} b^{n}=a^{i}(b a)^{j} a^{\ell-1}(b a) b^{k-1}(b a)^{m} b^{n}=a^{i+\ell-1}(b a)^{j+1+m} b^{k-1+n} .
$$

If $k$ and $\ell$ are even, then

$$
a^{i}(b a)^{j} b^{k} \cdot a^{\ell}(b a)^{m} b^{n}=a^{i}(b a)^{j} a^{\ell} b^{k}(b a)^{m} b^{n}=a^{i+\ell}(b a)^{j+m} b^{k+n} .
$$

If $k$ is even and $\ell$ is odd, then

$$
\begin{aligned}
& a^{i}(b a)^{j} b^{k} \cdot a^{\ell}(b a)^{m} b^{n}=a^{i}(b a)^{j} a^{\ell} b^{k}(b a)^{m} b^{n} \\
& =\widehat{\delta}_{j, 0} a^{i+\ell+1}(b a)^{j-1}\left(b^{k+1}(b a)^{m}\right) b^{n}+\delta_{j, 0} a^{i+\ell} b^{k}(b a)^{m} b^{n} \\
& =\widehat{\delta}_{j, 0}\left[\widehat{\delta}_{m, 0} a^{i+\ell+1}(b a)^{j-1} a(b a)^{m-1} b^{k+2+n}+\delta_{m, 0} a^{i+\ell+1}(b a)^{j-1} b^{k+n+1}\right] \\
& \quad+\delta_{j, 0} a^{i+\ell}(b a)^{m} b^{k+n} .
\end{aligned}
$$

Using (23) completes the proof. If $k$ is odd and $\ell$ is even, then

$$
\begin{aligned}
& a^{i}(b a)^{j} b^{k} a^{\ell}(b a)^{m} b^{n}=a^{i}(b a)^{j} a^{\ell} b^{k}(b a)^{m} b^{n}=a^{i+\ell}(b a)^{j} b^{k}(b a)^{m} b^{n} \\
& =\widehat{\delta}_{m, 0} a^{i+\ell}(b a)^{j} a(b a)^{m-1} b^{k+n+1}+\delta_{m, 0} a^{i+\ell}(b a)^{j} b^{k+n} .
\end{aligned}
$$

Using (23) again completes the proof.

4.6. The anti-Jordan family, $q=\infty$. The structure constants for $A^{\omega_{A J}^{\infty}}$ are

$$
\left[e_{1}, e_{1}, e_{2}\right]=-2 e_{1}, \quad\left[e_{2}, e_{1}, e_{1}\right]=2 e_{1}, \quad\left[e_{1}, e_{2}, e_{2}\right]=2 e_{2}, \quad\left[e_{2}, e_{2}, e_{1}\right]=-2 e_{2} .
$$

Proposition 4.26. The universal associative envelope $U\left(A^{\omega_{A J}^{\infty}}\right)$ is isomorphic to the down-up algebra $A(2,-1,-2)$, so we have $U\left(A^{\omega_{A J}^{\infty}}\right) \cong U\left(A_{L}^{\omega_{L}^{\infty}}\right)$.

Proof. Similar to the proof of Lemma 4.17.

4.7. The anti-Jordan family, $q=\frac{1}{2}$. The structure constants for $A^{\omega_{A J}^{1 / 2}}$ are zero.

Proposition 4.27. The universal associative envelope $U\left(A^{\omega_{A J}^{1 / 2}}\right)$ is isomorphic to the down-up algebra $A(0,1,0)$, so we have $U\left(A^{\omega_{A J}^{1 / 2}}\right) \cong U\left(A_{L}^{\omega_{L}^{1 / 2}}\right)$.

Proof. Similar to the proof of Lemma 4.22

Remark 4.28. The structure $A_{A J}^{\omega_{A J}^{1 / 2}}$ is an example of non-simple anti-Jordan triple systems. For the classification of simple finite-dimensional anti-Jordan triple systems see [2, Theorem 6]. For the universal associative envelope of the anti-Jordan triple system of all $n \times n$ matrices see [10, Theorem 6.2.12]. 


\section{Finite dimensional EnVElopes}

In this section, we consider the trilinear operations of "Jordan type".

5.1. The Jordan family, $q=\infty$. The structure constants for $A^{\omega_{J}^{\infty}}$ are

$$
\left[e_{1}, e_{2}, e_{1}\right]=2 e_{1}, \quad\left[e_{2}, e_{1}, e_{2}\right]=2 e_{2} .
$$

Theorem 5.1. A basis for $U\left(A^{\omega_{J}^{\infty}}\right)$ consists of the elements 1, $a, b, a b, b a$. The structure constants are $a \cdot b=a b, a \cdot b a=a, b \cdot a=b a, b \cdot a b=b, a b \cdot a=a, a b \cdot a b=a b$, $b a \cdot b=b, b a \cdot b a=b a$. The Wedderburn decomposition is $U\left(A^{\omega_{J}^{\infty}}\right)=\mathbb{Q} \oplus M_{2 \times 2}$. The only finite dimensional irreducible representations are the trivial 1-dimensional representation and the natural 2-dimensional representation.

Proof. We have $U=U\left(A^{\omega_{J}^{\infty}}\right)=F\langle a, b\rangle / I$ where $I$ is generated by $b^{3}, b^{2} a+a b^{2}$, $b a b-b, b a^{2}+a^{2} b, a b a-a, a^{3}$. We compute a Gröbner basis of $I$. There are four compositions with normal forms $a b^{2}, a^{2} b, b^{2}, a^{2}$. Including these with the original generators and self-reducing the resulting set produces the four generators $b a b-b, a b a-a, b^{2}, a^{2}$. All compositions of these elements reduce to 0 , and so we have a Gröbner basis. A basis for the quotient algebra consists of the cosets of the monomials which are not divisible by the leading monomial of any element of the Gröbner basis. This gives the stated basis for $U$. It follows that $U$ satisfies $a^{2}=0$, $b^{2}=0, a b a=a, b a b=b$ and these give the stated structure constants.

To decompose $U$ we follow [4. Using [4, Corollary 12] we verify that the radical is zero, and hence $U$ is semisimple. By 4, Corollary 15] the center $Z(U)$ has dimension 2 , basis $z_{1}=1, z_{2}=a b+b a$, and structure constants $z_{1} \cdot z_{1}=z_{1}, z_{1} \cdot z_{2}=z_{2}$, $z_{2} \cdot z_{2}=z_{2}$. Since $z_{2}^{2}=z_{2}$, the minimal polynomial of $z_{2}$ is $t^{2}-t$. Thus $Z(U)$ splits in two 1-dimensional ideals with bases $z_{2}-z_{1}$ and $z_{2}$. Scaling these basis elements to obtain idempotents gives $e_{1}=-z_{2}+z_{1}, e_{2}=z_{2}$. The corresponding elements in $U$ are $e_{1}=-a b-b a+1, e_{2}=a b+b a$. The ideals in $U$ generated by $e_{1}$ and $e_{2}$ have dimensions 1 and 4 respectively, and this gives the Wedderburn decomposition.

5.2. The Jordan family, $q=0$. The structure constants for $A^{\omega_{J}^{0}}$ are

$$
\left[e_{1}, e_{2}, e_{1}\right]=\left[e_{2}, e_{1}, e_{1}\right]=e_{1}, \quad\left[e_{2}, e_{1}, e_{2}\right]=\left[e_{1}, e_{2}, e_{2}\right]=e_{2} .
$$

Theorem 5.2. A basis for $U\left(A^{\omega_{J}^{0}}\right)$ consists of the elements $1, a, b, a^{2}, a b, b a$, $b^{2}, a b a, a b^{2}$. The structure constants are $a \cdot a=a^{2}, a \cdot b=a b, a \cdot b a=a b a$, $a \cdot b^{2}=a b^{2}, b \cdot a=b a, b \cdot b=b^{2}, b \cdot a^{2}=a-a b a, b \cdot a b=b-a b^{2}, b \cdot a b a=b a$, $b \cdot a b^{2}=b^{2}, a b \cdot a=a b a, a b \cdot b=a b^{2}, a b \cdot a^{2}=a^{2}, a b \cdot a b=a b, a b \cdot a b a=a b a$, $a b \cdot a b^{2}=a b^{2}, b a \cdot a=a-a b a, b a \cdot b=b-a b^{2}, b a \cdot b a=b a, b a \cdot b^{2}=b^{2}, a b a \cdot a=a^{2}$, $a b a \cdot b=a b, a b a \cdot b a=a b a, a b a \cdot b^{2}=a b^{2}$. The Wedderburn decomposition is $U\left(A^{\omega_{J}^{0}}\right)=\mathfrak{R} \oplus \mathbb{Q} \oplus M_{2 \times 2}$ where $\mathfrak{R}$ is the radical of dimension 4 . There are only two finite dimensional irreducible representations.

Proof. We have $U=U\left(A_{J}^{\omega_{J}^{0}}\right)=F\langle a, b\rangle / I$ where $I$ is generated by $b^{3}, b^{2} a, b a b+$ $a b^{2}-b, b a^{2}+a b a-a, a^{2} b, a^{3}$. This set is a Gröbner basis for $I$. Hence $U$ is finite dimensional and has the stated basis. The following relations hold in $U: b^{3}=0$, $b^{2} a=0, b a b=-a b^{2}+b, b a^{2}=-a b a+a, a^{2} b=0, a^{3}=0$. These imply the stated structure constants. Using [4, Corollary 12], a basis of the radical $\mathfrak{R}=\mathfrak{R}(U)$ consists of the elements $\xi_{1}=a-a b a, \xi_{2}=a^{2}, \xi_{3}=b^{2}, \xi_{4}=a b^{2}$. Hence we have these relations in $Q=U / \mathfrak{R}: a=a b a, a^{2}=b^{2}=a b^{2}=0$. The semisimple quotient $Q$ has dimension 5, and a basis consists of the cosets of $\eta_{1}=1, \eta_{2}=b, \eta_{3}=a b$, 
$\eta_{4}=b a, \eta_{5}=a b a$. The center $Z(Q)$ has dimension 2 , basis $z_{1}=\eta_{1}, z_{2}=\eta_{3}+\eta_{4}$, and structure constants $z_{1} \cdot z_{1}=z_{1}, z_{1} \cdot z_{2}=z_{2} \cdot z_{1}=z_{2}, z_{2} \cdot z_{2}=z_{2}$. Since $z_{2}^{2}=z_{2}$, the minimal polynomial of $z_{2}$ is $t^{2}-t$. Thus $Z(Q)=J \oplus K$ where $J=\left\langle z_{2}-z_{1}\right\rangle$ and $K=\left\langle z_{2}\right\rangle$ and both ideals are 1-dimensional. Scaling the basis elements to obtain idempotents gives $e_{1}=z_{1}-z_{2}, e_{2}=z_{2}$. The corresponding elements in $Q$ are $e_{1}=\eta_{1}-\eta_{3}-\eta_{4}, e_{2}=\eta_{3}+\eta_{4}$. The ideals in $Q$ generated by $e_{1}$ and $e_{2}$ have dimensions 1 and 4 respectively, and this gives the Wedderburn decomposition.

5.3. The Jordan family, $q=\frac{1}{2}$. The structure constants for $A^{\omega_{J}^{1 / 2}}$ are

$$
\begin{aligned}
& {\left[e_{1}, e_{1}, e_{2}\right]=\left[e_{1}, e_{2}, e_{1}\right]=\left[e_{2}, e_{1}, e_{1}\right]=2 e_{1},} \\
& {\left[e_{2}, e_{2}, e_{1}\right]=\left[e_{2}, e_{1}, e_{2}\right]=\left[e_{1}, e_{2}, e_{2}\right]=2 e_{2} .}
\end{aligned}
$$

Theorem 5.3. We have the isomorphism $U\left(A^{\omega_{J}^{1 / 2}}\right) \cong U\left(A^{\omega_{J}^{\infty}}\right)$.

Proof. We have $U=U\left(A^{\omega_{J}^{1 / 2}}\right)=F\langle a, b\rangle / I$ where $I$ is generated by $b^{3}, b^{2} a+\frac{1}{2} b a b-$ $\frac{1}{2} b, b^{2} a+2 b a b+3 a b^{2}-2 b, b a^{2}+\frac{2}{3} a b a+\frac{1}{3} a^{2} b-\frac{2}{3} a, a b a+2 a^{2} b-a, a^{3}$. The first iteration of the Gröbner basis algorithm produces the seven compositions $b a b^{2}-2 b^{2}$, $b a b^{2}-\frac{1}{2} b^{2}, b a b-b, b a b+2 a b^{2}-b, b a b+\frac{3}{2} a b^{2}-b, a^{2} b, a^{2}$. Including these with the original generators, and self-reducing the resulting set, produces the same ideal generators as for $q=\infty$; hence the two quotient algebras are isomorphic.

5.4. The Jordan family, $q=1$. The structure constants for $A^{\omega_{J}^{1}}$ are

$$
\left[e_{1}, e_{1}, e_{2}\right]=\left[e_{1}, e_{2}, e_{1}\right]=e_{1}, \quad\left[e_{2}, e_{2}, e_{1}\right]=\left[e_{2}, e_{1}, e_{2}\right]=e_{2} .
$$

Theorem 5.4. A basis for $U\left(A^{\omega_{J}^{1}}\right)$ consists of the elements $1, a, b, a^{2}, a b, b a, b^{2}$, $a^{2} b, b a b$. The structure constants of $U\left(A^{\omega_{J}^{1}}\right)$ are $a \cdot a=a^{2}, a \cdot b=a b, a \cdot a b=a^{2} b$, $a \cdot b a=a-a^{2} b, a \cdot b a b=a b, b \cdot a=b a, b \cdot b=b^{2}, b \cdot a b=b a b, b \cdot b a=b-b a b$, $b \cdot b a b=b^{2}, a^{2} \cdot b=a^{2} b, a^{2} \cdot b a=a^{2}, a^{2} \cdot b a b=a^{2} b, a b \cdot a=a-a^{2} b, a b \cdot a b=a b$, $b a \cdot b=b a b, b a \cdot b a=b a, b a \cdot b a b=b a b, b^{2} \cdot a=b-b a b, b^{2} \cdot a b=b^{2}, a^{2} b \cdot a=a^{2}$, $a^{2} b \cdot a b=a^{2} b, b a b \cdot a=b a, b a b \cdot a b=b a b$. The Wedderburn decomposition is $U\left(A^{\omega_{J}^{1}}\right)=\mathfrak{R} \oplus \mathbb{Q} \oplus M_{2 \times 2}$ where $\mathfrak{R}$ is the radical of dimension 4. There are two finite dimensional irreducible representations.

Proof. The original set of generators of the ideal $I$ is a Gröbner basis and consists of the six elements $b^{3}, b^{2} a+b a b-b, b a^{2}, a b^{2}, a b a+a^{2} b-a, a^{3}$. Hence $U=U\left(A^{\omega_{J}^{1}}\right)$ is finite dimensional with the stated basis. The following relations hold in $U: b^{3}=0$, $b^{2} a=-b a b+b, b a^{2}=0, a b^{2}=0, a b a=-a^{2} b+a, a^{3}=0$. These give the stated structure constants. A basis of the radical $\mathfrak{R}=\mathfrak{R}(U)$ consists of the elements $\xi_{1}=b-b a b, \xi_{2}=a^{2}, \xi_{3}=b^{2}, \xi_{4}=a^{2} b$ which give these relations in $Q=U / \mathfrak{R}$ : $b=b a b, a^{2}=b^{2}=a^{2} b=0$. The semisimple quotient $Q$ has dimension 5 and a basis consists of the cosets of $\eta_{1}=1, \eta_{2}=a, \eta_{3}=a b, \eta_{4}=b a, \eta_{5}=b a b$. The center $Z(Q)$ has dimension 2 with basis $z_{1}=\eta_{1}, z_{2}=\eta_{3}+\eta_{4}$ and structure constants $z_{1} \cdot z_{1}=z_{1}, z_{1} \cdot z_{2}=z_{2} \cdot z_{1}=z_{2}, z_{2} \cdot z_{2}=z_{2}$. Since $z_{2}^{2}=z_{2}$, the minimal polynomial of $z_{2}$ is $t^{2}-t$. Thus $Z(Q)=J \oplus K$, where $J=\left\langle z_{2}-z_{1}\right\rangle$ and $K=\left\langle z_{2}\right\rangle$; both ideals are 1-dimensional. Scaling these basis elements to obtain idempotents gives $e_{1}=z_{1}-z_{2}, e_{2}=z_{2}$. The corresponding elements of $Q$ are $e_{1}=\eta_{1}-\eta_{3}-\eta_{4}$, $e_{2}=\eta_{3}+\eta_{4}$. The ideals in $Q$ generated by $e_{1}$ and $e_{2}$ have dimensions 1 and 4 respectively, and this gives the Wedderburn decomposition. 
5.5. The anti-Jordan family, $q=-1$. The structure constants for $A^{\omega_{A J}^{-1}}$ are

$$
\left[e_{1}, e_{1}, e_{2}\right]=-e_{1}, \quad\left[e_{1}, e_{2}, e_{1}\right]=e_{1}, \quad\left[e_{2}, e_{1}, e_{2}\right]=e_{2}, \quad\left[e_{2}, e_{2}, e_{1}\right]=-e_{2} .
$$

Theorem 5.5. We have the isomorphisms $U\left(A^{\omega_{A J}^{-1}}\right) \cong U\left(A^{\omega_{J}^{1 / 2}}\right) \cong U\left(A^{\omega_{J}^{\infty}}\right)$.

Proof. We have $U=U\left(A^{\omega_{A J}^{-1}}\right)=F\langle a, b\rangle / I$ where $I$ is generated by $b^{2} a-b a b+b$ and $a b a-a^{2} b-a$. The first iteration of the Gröbner basis algorithm produces one composition, $b a b-b$. Including this element with the original generators, and self-reducing the resulting set, produces a new set of three generators: $b^{2} a$, $b a b-b, a b a-a^{2} b-a$. The second iteration produces three compositions: $b a^{2} b$, $a^{2} b^{2}, b^{2}$. Including these elements with the previous generators, and self-reducing the resulting set, produces a new set of four generators: $b a^{2} b, b a b-b, a b a-a^{2} b-a$, $b^{2}$. The third iteration produces two compositions: $b a^{3} b+b a^{2}, a^{2} b$. Including these elements with the previous generators, and self-reducing the resulting set, produces a new set of five generators: $b a b-b, b a^{2}, a b a-a, a^{2} b, b^{2}$. The fourth iteration produces one composition, $a^{2}$. Including this element with the previous generators, and self-reducing the resulting set, produces a new set of four generators: $b a b-b$, $a b a-a, b^{2}, a^{2}$. This is a Gröbner basis for the ideal, and is the same Gröbner basis as for the Jordan cases $q=\infty, q=\frac{1}{2}$; hence the quotient algebras are isomorphic.

5.6. The anti-Jordan family, $q=2$. The structure constants for $A^{\omega_{A J}^{2}}$ are

$$
\left[e_{1}, e_{2}, e_{1}\right]=e_{1}, \quad\left[e_{2}, e_{1}, e_{1}\right]=-e_{1}, \quad\left[e_{2}, e_{1}, e_{2}\right]=e_{2}, \quad\left[e_{1}, e_{2}, e_{2}\right]=-e_{2} .
$$

Proposition 5.6. We have $U\left(A^{\omega_{A J}^{2}}\right) \cong U\left(A^{\omega_{A J}^{-1}}\right)$.

Proof. Similar to the proof of Theorem 5.5

5.7. The last nine operations. We first consider the fourth family with $q=\infty$. The structure constants for $A^{\omega_{F}^{\infty}}$ are

$$
\begin{array}{ll}
{\left[e_{1}, e_{1}, e_{2}\right]=\left[e_{2}, e_{1}, e_{1}\right]=-e_{1}, \quad\left[e_{1}, e_{2}, e_{1}\right]=e_{1},} \\
{\left[e_{2}, e_{2}, e_{1}\right]=\left[e_{1}, e_{2}, e_{2}\right]=-e_{2}, \quad\left[e_{2}, e_{1}, e_{2}\right]=e_{2} .}
\end{array}
$$

Proposition 5.7. We have $U\left(A^{\omega_{F}^{\infty}}\right) \cong U\left(A^{\omega_{J}^{\infty}}\right)$.

Proof. We have $U\left(A^{\omega_{F}^{\infty}}\right)=F\langle a, b\rangle / J$ where $J$ is generated by $b^{3}, b^{2} a-b a b+a b^{2}+b$, $b a b-b, b a^{2}-a b a+a^{2} b+a, a b a-a, a^{3}$. Self-reducing this set of generators gives the set of generators for the Jordan case, $q=\infty$ (see the proof of Theorem 5.1).

For the fourth family with $q=0$, the structure constants for $A^{\omega_{F}^{0}}$ are

$$
\left[e_{1}, e_{2}, e_{1}\right]=e_{1}, \quad\left[e_{2}, e_{1}, e_{2}\right]=e_{2} .
$$

Proposition 5.8. We have $U\left(A^{\omega_{F}^{0}}\right) \cong U\left(A^{\omega_{J}^{0}}\right)$.

Proof. Similar to the proof of Proposition 5.7

For the fourth family with $q=1$, the structure constants for $A^{\omega_{F}^{1}}$ are

$$
\left[e_{1}, e_{2}, e_{1}\right]=e_{1}, \quad\left[e_{2}, e_{1}, e_{2}\right]=e_{2} .
$$

Proposition 5.9. We have $U\left(A^{\omega_{F}^{1}}\right) \cong U\left(A^{\omega_{J}^{1}}\right)$.

Proof. Similar to the proof of Proposition 5.7. 
We consider the last six operations together.

Fourth family, $q=-1$ : The structure constants for $A^{\omega_{F}^{-1}}$ are

$$
\left[e_{1}, e_{2}, e_{1}\right]=e_{1}, \quad\left[e_{2}, e_{1}, e_{1}\right]=2 e_{1}, \quad\left[e_{2}, e_{1}, e_{2}\right]=e_{2}, \quad\left[e_{1}, e_{2}, e_{2}\right]=2 e_{2} .
$$

Fourth family, $q=2$ : The structure constants for $A^{\omega_{F}^{2}}$ are

$$
\left[e_{1}, e_{1}, e_{2}\right]=2 e_{1}, \quad\left[e_{1}, e_{2}, e_{1}\right]=e_{1}, \quad\left[e_{2}, e_{2}, e_{1}\right]=2 e_{2}, \quad\left[e_{2}, e_{1}, e_{2}\right]=e_{2} .
$$

Fourth family, $q=\frac{1}{2}$ : The structure constants for $A^{\omega_{F}^{1 / 2}}$ are

$$
\begin{aligned}
& {\left[e_{1}, e_{1}, e_{2}\right]=\left[e_{1}, e_{2}, e_{1}\right]=\left[e_{2}, e_{1}, e_{1}\right]=e_{1},} \\
& {\left[e_{2}, e_{2}, e_{1}\right]=\left[e_{2}, e_{1}, e_{2}\right]=\left[e_{1}, e_{2}, e_{2}\right]=e_{2} .}
\end{aligned}
$$

Cyclic commutator: The structure constants for $A^{\omega_{c c}}$ are

$$
\left[e_{1}, e_{1}, e_{2}\right]=-e_{1}, \quad\left[e_{1}, e_{2}, e_{1}\right]=e_{1}, \quad\left[e_{2}, e_{2}, e_{1}\right]=-e_{2}, \quad\left[e_{2}, e_{1}, e_{2}\right]=e_{2} .
$$

Weakly commutative operation: The structure constants for $A^{\omega_{w c}}$ are

$$
\begin{array}{llll}
{\left[e_{1}, e_{1}, e_{2}\right]=-e_{1},} & {\left[e_{1}, e_{2}, e_{1}\right]=e_{1},} & {\left[e_{2}, e_{1}, e_{1}\right]=2 e_{1},} \\
{\left[e_{2}, e_{2}, e_{1}\right]=-e_{2},} & {\left[e_{2}, e_{1}, e_{2}\right]=e_{2},} & {\left[e_{1}, e_{2}, e_{2}\right]=2 e_{2} .}
\end{array}
$$

Weakly anti-commutative operation: The structure constants for $A^{\omega_{w a}}$ are

$$
\begin{array}{lll}
{\left[e_{1}, e_{2}, e_{1}\right]=\left[e_{1}, e_{1}, e_{2}\right]=e_{1},} & {\left[e_{2}, e_{1}, e_{1}\right]=-2 e_{1},} \\
{\left[e_{2}, e_{1}, e_{2}\right]=\left[e_{2}, e_{2}, e_{1}\right]=e_{2},} & {\left[e_{1}, e_{2}, e_{2}\right]=-2 e_{2} .}
\end{array}
$$

Proposition 5.10. We have the following isomorphisms:

$$
U\left(A^{\omega_{F}^{-1}}\right) \cong U\left(A^{\omega_{F}^{2}}\right) \cong U\left(A^{\omega_{F}^{1 / 2}}\right) \cong U\left(A^{\omega_{c c}}\right) \cong U\left(A^{\omega_{w c}}\right) \cong U\left(A^{\omega_{w a}}\right) \cong U\left(A^{\omega_{J}^{\infty}}\right) .
$$

Proof. We have $U\left(A^{\omega_{F}^{-1}}\right)=F\langle a, b\rangle / I$ where $I$ is generated by $b^{3}, b^{2} a+b a b+a b^{2}-b$, $b^{2} a+\frac{1}{2} a b^{2}, b a b+\frac{1}{2} a b^{2}-b, b a^{2}+2 a b a-2 a, b a^{2}+a b a+a^{2} b-a, b a^{2}+2 a^{2} b, a^{3}$. We compute a Gröbner basis for $I$. The first iteration produces eight compositions with the normal forms $a^{2} b^{2}, a^{2} b a-a^{2}, a^{2} b a, a b a-a^{2} b-a, a b^{2}, a^{2} b, b^{2}, a^{2}$. Including these elements with the original generators, and then self-reducing the resulting set, produces a new set of four ideal generators: $b a b-b, a b a-a, a^{2}, b^{2}$. This is a Gröbner basis $I$. In fact, this is the same Gröbner basis as in the Jordan case, $q=\infty$ (see the proof of Theorem [5.1). The other cases are similar.

\section{ACKNOWLEDGEMENTS}

The author thanks the anonymous referee for helpful comments. This work forms part of the author's doctoral thesis. The author was supported by a Teacher Scholar Doctoral Fellowship from the University of Saskatchewan. She thanks her supervisor, Prof. Murray Bremner, for many suggestions and insights which greatly improved this paper, and Prof. Chris Soteros for the reference to [22, Example 10.3].

\section{REFERENCES}

[1] G. M. Bergman: The diamond lemma for ring theory. Adv. Math. 29 (1978) 178-218.

[2] S. Bashir: Automorphisms of Simple Anti-Jordan Pairs. Ph.D. thesis, University of Ottawa, 2008.

[3] G. Benkart, T. Roby: Down-up algebras. J. Algebra 209 (1998) 305-344.

[4] M. R. Bremner: How to compute the Wedderburn decomposition of a finite dimensional associative algebra. Groups Complex. Cryptol 3 (2011) 47-66. 
[5] M. R. Bremner, L. A. Peresi: Classification of trilinear operations. Comm. Algebra 35 (2007) 2932-2959.

[6] T. Cassidy, B. Shelton: Basic properties of generalized down-up algebras. J. Algebra 279 (2004), no. 1, 402-421.

[7] V. Chari, A. Pressley: A Guide to Quantum Groups. Corrected reprint of the 1994 original. Cambridge University Press, Cambridge, 1995. xvi+651 pp.

[8] W. A. De Graff: Lie Algebras: Theory and Algorithms. North-Holland, Amsterdam, 2000.

[9] H. A. Elgendy, M. R. Bremner: Universal associative envelopes of $(n+1)$-dimensional $n$-Lie algebras. Comm. Algebra 40 (2012), no. 5, 1827-1842.

[10] H. A. Elgendy: Polynomial Identities and Enveloping Algebras for n-ary Structures. Ph.D. thesis, University of Saskatchewan, 2012.

[11] J. Faraut, S. Kaneyuki, A. Koranyi, Q.-K. Lu, G. Roos: Analysis and Geometry on Complex Homogeneous Domains. Progress in Mathematics, 185. Birkhauser Boston, Inc., Boston, MA, 2000.

[12] H. Freudenthal: Lie groups in the foundations of geometry. Advances in Math. 11964 fasc. 2, 145-190(1964).

[13] T. L. Hodge, B. J. Parshall: On the representations theory of Lie triple systems. Trans. Amer. Math. Soc. 354(2002), no. 11, 4359-4391.

[14] J. E. Humphreys: Introduction to Lie Algebras and Representation Theory. Springer, New York, 1972.

[15] N. Jacobson: Structure and Representations of Jordan Algebras. American Mathematical Society, Providence, 1968.

[16] N. KamiYa, S. Oкubo: On triple systems and Yang-Baxter equations. Proceedings of the Seventh International Colloquium on Differential Equations (Plovdiv, 1996), 189-196, VSP, Utrecht, 1997.

[17] W. G. Lister: Ternary rings. Trans. Amer. Math. Soc 154 (1971) 37-55.

[18] O. Loos: Symmetric spaces. I: General theory. W. A. Benjamin, Inc., New York-Amsterdam 1969 viii+198 pp. $22.70(53.00)$

[19] O. Loos: Lectures on Jordan Triples. The University of British Columbia, Vancouver, B.C., 1971. iii+67 pp.

[20] K. Meyberg: Lectures on Algebras and Triple Systems. Notes on a course of Lectures given during the academic year 1971-1972. The University of Virgina, Charlottesville, Va., 1972. $\mathrm{v}+226 \mathrm{pp}$.

[21] B. Rosenfeld: Geometry of Lie Groups. Mathematics and its Applications, 393. Kluwer Academic Publishers Group, Dordrecht, 1997. xviii+393 pp.

[22] J. H. van Lint, R. M. Wilson: A Course in Combinatorics. Cambridge University Press, 1992.

[23] K. ZhaO: Centers of down-up algebras. J. Algebra 214 (1999) 103-121.

Department of Mathematics and Statistics, University of Saskatchewan, Canada

E-mail address: hae431@mail.usask.ca 